\title{
Colloquium: The glass transition and elastic models of glass-forming liquids
}

\author{
Jeppe C. Dyre \\ Department of Mathematics and Physics (IMFUFA), DNRF Centre "Glass and Time," \\ Roskilde University, Postbox 260, DK-4000 Roskilde, Denmark
}

(Published 29 September 2006)

\begin{abstract}
Basic characteristics of the liquid-glass transition are reviewed, emphasizing its universality and briefly summarizing the most popular phenomenological models. Discussion is focused on a number of alternative models which one way or the other connect the fast and slow degrees of freedom of viscous liquids. It is shown that all these "elastic" models are equivalent in the simplest approximation.
\end{abstract}

DOI: 10.1103/RevModPhys.78.953

PACS number(s): 64.70.Pf, 62.10.+s, 62.20.Dc

\section{CONTENTS}

I. Introduction

II. Glass Formation and the Three Non's
A. The glass transition
B. The three non's
C. Kauzmann's paradox

III. Models of the Conventional Wisdom
A. Entropy model
B. Free-volume models
C. Other models

IV. Elastic Models: Connecting the Fast and Slow Degrees of Freedom
A. Harmonic approximation
B. Models of Mooney and Bueche
C. Nemilov's approach
D. Shoving model
E. How do elastic models compare to experiment?

V. Common Features of Elastic Models
A. Equivalence of elastic models in the simplest approximation
B. The thermodynamic connection
C. Relation to point-defect thermodynamics and solid-state diffusion

VI. Recent Developments

VII. Outlook

Acknowledgments

References

\section{INTRODUCTION}

The earliest glaze known is that on stone beads of the Badarian age in Egypt, about 12000 B.C. Green glaze was applied to powdered quartz for making small figures about 9000 B.C. The oldest pure glass is a moulded amulet of deep lapis lazuli color, of about 7000 B.C. (Petrie, 1925). Although glass is the oldest artificial material utilized by man, new discoveries and applications continue to appear. Thus the glassy state of pharmaceuticals is being recognized as more effective than the crystalline form because it dissolves more quickly; in that regard, the glassy state of a common medicine, aspirin, has been recently studied (Johari and Pyke, 2000). Only in the 1980s was it realized how important the glass transition is in providing stability to otherwise labile materials and that the glass transition plays a central role in the preservation of food (Le Meste et al., 2002; Levine, 2002; Kasapis, 2005). Traditional applications of glasses and glass science include, e.g., optical fibers or glass ceramics - and of course glass is still extensively used for windows and containers, not to mention the beautiful artworks based on a thousand-year old tradition.

A glass is formed by cooling a liquid fast enough to avoid crystallization. At continued supercooling the liquid viscosity increases dramatically, and at some point the liquid freezes continuously into a noncrystalline solid. This is termed the glass transition, although it is not a phase transition with a well-defined transition temperature (Brawer, 1985; Angell, 1991; Debenedetti, 1996).

Many liquids require fast cooling to avoid crystallization, e.g., most alloys, but there are also many liquids that are easily supercooled and, in fact, difficult to crystallize, e.g., silicates and numerous organic liquids. Any liquid is able to form a glass if cooled rapidly enough (Tammann, 1925). In view of this universality, the glassy state may be regarded as the fourth state of conventional matter: Glass is solid as is the crystalline state, but isotropic and without long-range order as is the liquid state. This unique combination of properties explains the immense importance of glasses for a variety of applications (Johari, 1974). Indeed, it is glass' lack of long-range order-rather than the traditional properties of transparency, brittleness, and low electrical conductivitythat is the defining characteristic of this type of material (Cotterill, 1985).

The glass transition was unknown to most physicists until a few decades ago, and the glassy state was barely mentioned in textbooks on condensed-matter physics. The focus on amorphous semiconductors and spin glasses of the 1980s led to an increasing interest in glasses and glass formation. Glassy dynamics of various kinds showed up repeatedly in quite different contexts, so why not study the real thing? In the 1990s the re- 
search field "viscous liquids and the glass transition" grew into an accepted branch of condensed-matter physics, catalyzed by the increasing realization that fundamental problems remain unsolved.

After giving an overview of the basic experimental facts and prevailing models, this Colloquium focuses on an alternative approach to solving a central problem of the field: How to explain the often dramatic temperature dependence of the relaxation time of glass-forming liquids. This alternative approach, which connects the fast and slow degrees of freedom, involves several related "elastic" models. The final section outlines some consequences if elastic models are correct.

\section{GLASS FORMATION AND THE THREE NON'S}

\section{A. The glass transition}

Figure 1(a) shows the specific volume of selenium as a function of temperature during cooling. The gradual shrinking of the liquid continues unaffected by the freezing temperature $T_{m}$. At some point the expansion coefficient - the slope of the curve-decreases to a value close to that of the crystalline state. This is the glass transition, which takes place at a slightly lower temperature if the cooling is slower. A similar observation is made for the enthalpy. Figure 1(b) shows a schematic drawing of the enthalpy during cooling and subsequent reheating. The glass transition is continuous and cooling-rate dependent, and there is hysteresis upon reheating.

The glass transition is similar to a second-order phase transition in the Ehrenfest sense with continuity of volume and entropy, but discontinuous changes of their derivatives (Goldenfeld, 1992). But the transition is continuous and cooling-rate dependent, so it cannot be a genuine phase transition. What is going on? A clue is provided by the fact that the viscosity of a liquid approaching the glass transition always becomes extremely large.

Recall the definition of viscosity $\eta$ : When a liquid is placed between two parallel solid plates of area $A$ moving with velocity $v$ relative to one another, the force $F$ needed to sustain the motion is given by $F=\eta v A / d$, where $d$ is the distance between the plates. The viscosity of ambient water is $10^{-3} \mathrm{~Pa}$ s. For a glass-forming liquid the viscosity is typically of order $10^{12} \mathrm{~Pa}$ just above the glass transition if the liquid is cooled by a rate of a few Kelvin per minute (this defines the "calorimetric" glass transition, henceforth just referred to as the glass transition). This viscosity, incidentally, is so large that conventional methods for measuring the viscosity completely fail. To appreciate such high viscosities, let us estimate how long a time $t$ it takes to empty a cup containing a liquid just above its glass transition: Substituting $v \sim l / t$, $F \sim 10 \mathrm{~N}$, and $A / d \sim l \sim 0.1 \mathrm{~m}$ into the definition of viscosity, one finds that $t \sim 10^{9} \mathrm{~s}$ (roughly 30 years). Such a system appears absolutely solid, but is still a liquid according to any reasonable scientific definition as long as it is in thermal equilibrium.
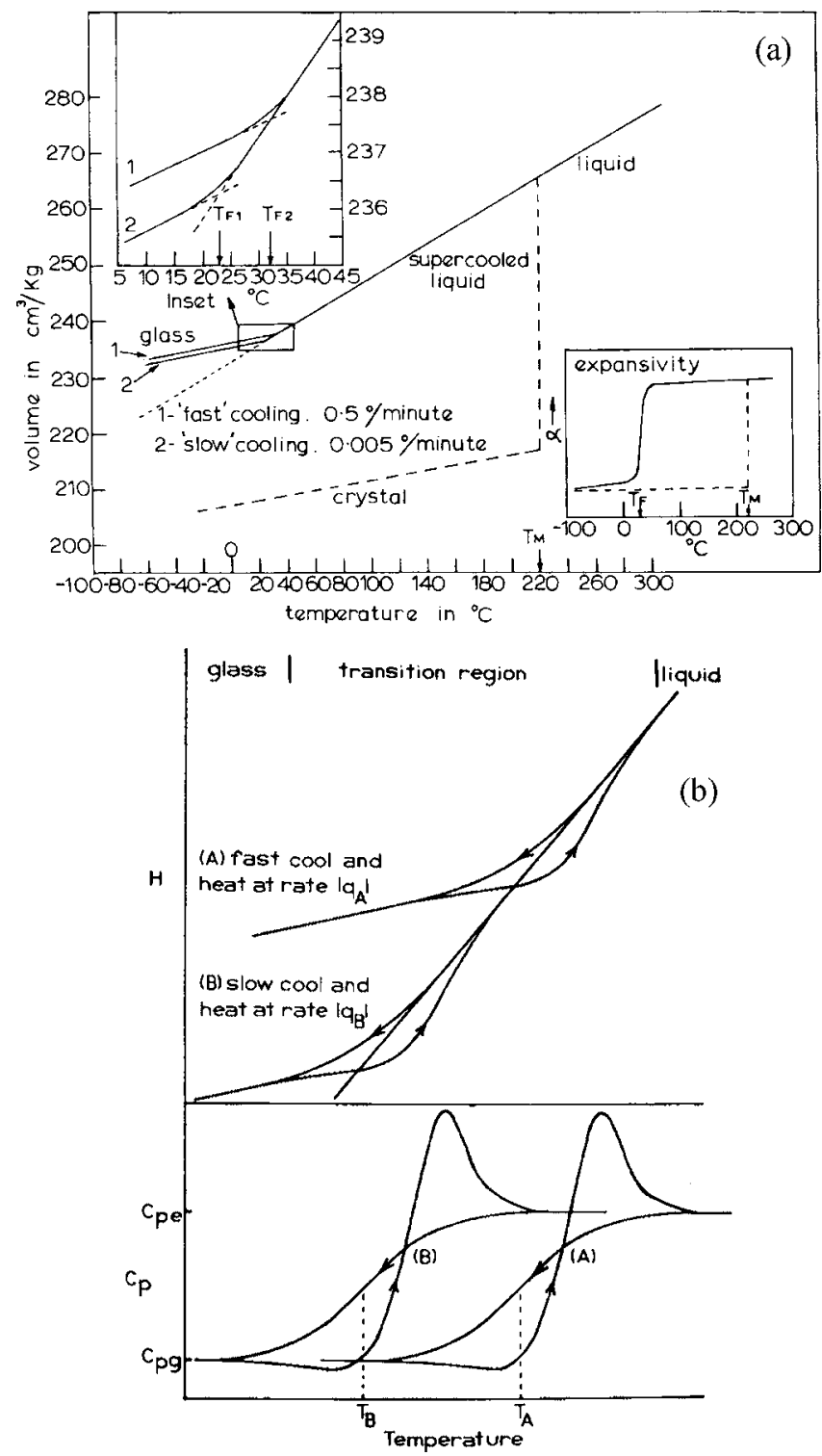

FIG. 1. Thermodynamic characteristics of the glass transition. (a) Volume of selenium measured during cooling from the liquid phase. Around $30{ }^{\circ} \mathrm{C}$ the expansivity decreases to a value close to that of the crystalline state; this is the glass transition. At slower cooling the glass transition takes place at a slightly lower temperature [adapted from Owen, 1985 (using data of Dzhalilov and Rzaev, 1967) with permission of Springer Science and Business Media]. (b) Schematic drawing of the enthalpy $H$ and specific heat $C_{P}$ by cooling from the liquid phase and subsequently reheating (the enthalpy is calculated by integrating the specific heat with respect to $T$ ). On reheating, the enthalpy follows a different path. Reprinted with permission from Moynihan et al., 1974. Copyright 1974 American Chemical Society.

A system falls out of equilibrium when its relaxation time is so long that it cannot equilibrate within a given time. Because of its dependence on gravity and sample size the above calculation cannot tell us anything about the equilibration time. The genuine "bulk" relaxation time $\tau$ of a liquid was first identified by Maxwell (1867). He suggested that on a sufficiently short time scale any 
liquid is elastic and behaves like a solid (Lamb, 1978). If $x$ is the relative displacement of the plates, the shear displacement $\gamma$ is defined as $\gamma \equiv x / d$; for an elastic solid the shear modulus $G$ is defined by $\sigma=G \gamma$, where $\sigma$ $\equiv F / A$ is the so-called shear stress. In terms of $\sigma$ and $\dot{\gamma}$ (the time derivative of $\gamma$ ) the definition of viscosity is $\sigma=\eta \dot{\gamma}$. Maxwell proposed extrapolating between liquid and solid behavior by assuming

$$
\dot{\gamma}=\frac{\sigma}{\eta}+\frac{\dot{\sigma}}{G} \text {. }
$$

Clearly, Eq. (1) applies for liquids in a steady-state flow $(\dot{\sigma}=0)$ as well as for solids $(\eta=\infty)$.

Consider a sudden shearing displacement starting from equilibrium, $\dot{\gamma}(t)=\gamma_{0} \delta(t)$. By integrating Eq. (1) one finds that immediately after $t=0$ one has $\sigma=G \gamma_{0}$. This suggests that $G$ should be termed the "instantaneous" or "infinite-frequency" shear modulus, usually denoted by $G_{\infty}$. Since $\dot{\gamma}=0$ for $t>0$, the stress subsequently decays exponentially to zero with relaxation time $\tau$ given by

$$
\tau=\frac{\eta}{G_{\infty}} .
$$

It is a general property of Eq. (1) that the liquid is solidlike on time scales much shorter than $\tau$-generic liquid behavior is only predicted on time scales much longer than $\tau$.

The Maxwell relaxation time $\tau$ provides the key to understanding the glass transition. Typical values of $G_{\infty}$ are in the $10^{9}-10^{10} \mathrm{~Pa}$ s range, so since $\eta \sim 10^{12} \mathrm{~Pa}$ at the glass transition, $\tau$ is here of order $100-1000 \mathrm{~s}$. Thus the glass transition takes place when the Maxwell relaxation time becomes comparable to the cooling time. Noting that independent of the unit system $d \ln T$ is the relative temperature change $d T / T$, the glass transition temperature $T_{g}$ is determined by

$$
\left|\frac{d \ln T}{d t}\right|_{T_{g}} \sim \frac{1}{\tau\left(T_{g}\right)} .
$$

The Maxwell relaxation time not only determines how fast a macroscopic stress relaxes. Numerous experiments show that $\tau$ also determines the typical time between molecular displacements or reorientations. This confirms that the glass transition takes place when the liquid is unable to equilibrate on the experimental time scale, i.e., when "some process in the amorphous material occurs too slowly at low temperatures to permit thermodynamic equilibrium to be established in all degrees of freedom" (Kauzmann, 1948).

What happens at the glass transition is that molecular motion virtually ceases-except for thermal vibrations ${ }^{1}$ (Simon, 1930, 1931; Tammann, 1933; Kauzmann, 1948; Johari, 1974; Harrison, 1976; Brawer, 1985; Scherer,

\footnotetext{
${ }^{1}$ Actually, minor motions often remain in the glassy phase via one or more so-called Johari-Goldstein (1970) beta processes, but these are unable to induce flow or relieve a macroscopic stress.
}

TABLE I. Examples of good glass formers with different chemical bonds. By definition, a good glass former is a liquid with very low rates of crystal nucleation and growth at all temperatures.

\begin{tabular}{lr}
\hline Glass-forming liquid & Chemical bond \\
\hline Silicates, borates & Covalent \\
$\mathrm{KNO}_{3}-\mathrm{Ca}\left(\mathrm{NO}_{3}\right)_{2}$ mixtures & Ionic \\
Ortho-terphenyl & van der Waals \\
Glycerol, glycose & Hydrogen \\
Pd-Cu-Ni-P alloys, Cu-Zr alloys & Metallic \\
\hline \hline
\end{tabular}

1986; Nemilov, 1995; Dyre, 1998; Angell et al., 2000; Alba-Simionesco, 2001; Debenedetti and Stillinger, 2001; Donth, 2001; Franks, 2003). This explains several observations:

- The ability to form glasses is universal and not a specific atomic or molecular property-once the extremely viscous liquid state has been arrived at, glass formation is unavoidable upon continued cooling.

- Volume, enthalpy, and entropy are continuous across the glass transition, and no changes of the molecular structure are observed at $T_{g}$.

- The glass expansion coefficient and specific heat are lower than those of the liquid - this is because below $T_{g}$ molecular reorientations and effective motions cease to contribute to these quantities.

- $T_{g}$ is lower when the cooling rate is reduced (in fact $T_{g}$ has no exact definition, even for a given cooling rate).

- Hysteresis effects are inherently associated with the glass transition- these derive from the fact that upon reheating the structure relaxes slightly before $T_{g}$ is reached.

The universality of the property to form glasses was suggested long ago (Tammann, 1925, 1933; Simon, 1931), but even many years thereafter the broader scientific community tended to believe that, as for silicates, glasses primarily form from covalently bonded network liquids. Glasses may form from all liquids, however, with liquid helium as the outstanding exception. In many cases, e.g., most metallic liquids, a rapid cooling is necessary to avoid crystallization (cooling rates $\sim 10^{6} \mathrm{~K} / \mathrm{s}$ ). A "good glass former" is a liquid which is readily supercooled, i.e., characterized by very low rates of crystal nucleation and growth at all temperatures (Angell, 1995; Pimpinelli and Villain, 1998). Examples of good glass formers are given in Table I; note that there are now also good metallic glass formers, the so-called bulk metallic glasses (Löffler, 2003; Wang, Dong, and Shek, 2004).

Figure 2 shows the radial distribution function $g(r)$ for liquid and glassy selenium. There is little difference, although the liquid data were taken far above $T_{g} \cong 300 \mathrm{~K}$; the glass simply inherits the liquid structure and its lack 

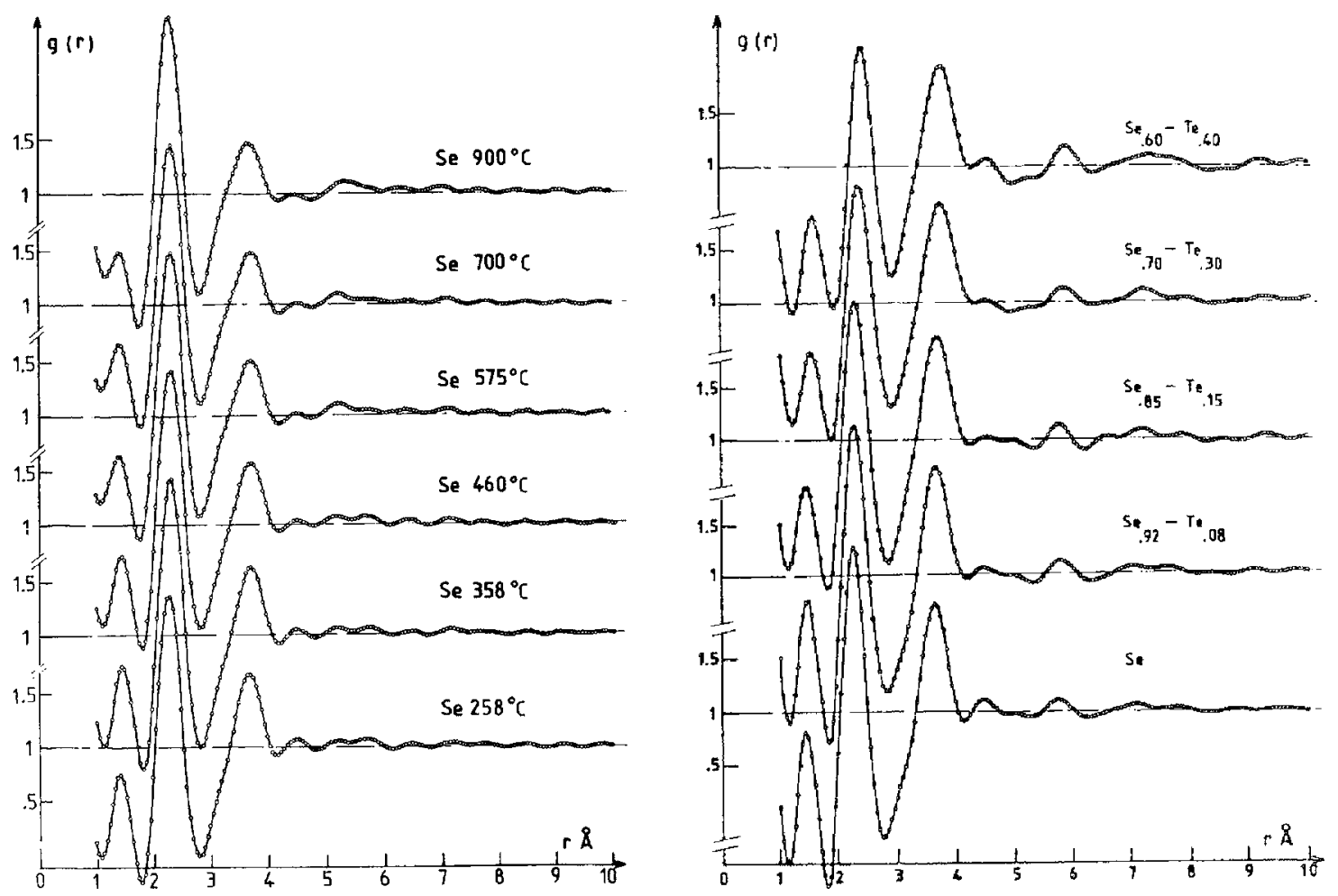

FIG. 2. Radial distribution function for liquid selenium (left) and glassy selenium-tellurium mixtures (right) measured by neutron scattering. Left: There are only moderate changes upon cooling although the viscosity increases by many orders of magnitude in this temperature range. Right: The radial distribution function of glassy selenium (bottom) is not much different from that of liquid selenium. Reprinted from Bellissent and Tourand, 1980. Copyright 1980, with permission from Elsevier.

of long-range order. Generally, solids without long-range order are termed amorphous. Amorphous solids may be produced by a variety of methods like vapor deposition, sputtering, solution hydrolysis, bombardment of crystals with high-energy particles, milling of crystals, etc. (Elliott, 1990). The term "glass" is traditionally reserved for amorphous solids produced by cooling a liquid, ${ }^{2}$ although this convention was criticized by Doremus (1994) on the grounds that solids should be named after their properties, not how they are produced.

The glass transition may be regarded as an artifact due to our inability to do experiments slow enough to allow the molecules to change their configurations (Johari, 1974). Simon $(1930,1931)$ first emphasized that, because glass is an out-of-equilibrium liquid, any glass continuously approaches the liquid state. Thus glass properties change with time, at least in principle, and there is no unique glassy state. The latter is also reflected by the fact that glass disobeys the third law of thermodynamics by having a positive entropy at zero temperature. The relaxation of glass properties is

\footnotetext{
${ }^{2}$ An amorphous solid may also be produced by keeping the liquid at constant temperature and raising the pressure. Such a solid would usually also be termed a glass; it could be brought to ambient pressure by lowering the temperature while keeping the pressure high, and subsequently relieving the pressure. The resulting glass differs from one produced by cooling at ambient pressure.
}

termed "annealing" or "stabilization" when it is controlled and "physical aging" or just "aging" when it is unwanted. Unless the glass is produced by very fast cooling, however, glass relaxation can only be observed just below $T_{g}$.

\section{B. The three non's}

Most applications of glass science deal with the glassy state or relaxation just below the glass transition. In contrast, much of the scientific focus is on the viscous liquid phase preceding glass formation. One reason for this is that glasses derive from liquids, and just as in social sciences or history one must know the past to understand the present. Moreover, even basic properties of viscous liquids are not well understood.

How are viscous liquids studied? Techniques like neutron, x-ray, or light scattering are utilized for studying both structure and dynamics (Hansen and McDonald, 1986). NMR is a useful tool for assessing, in particular, dynamical properties beyond those entailed in two-point correlation functions (Böhmer et al., 2001). Important macroscopic observables include the frequencydependent dielectric constant (Kudlik et al., 1999; Lunkenheimer et al., 2000; Kremer and Schönhals, 2002), specific heat (Birge and Nagel, 1985; Christensen, 1985), compressibility (Christensen and Olsen, 1995), and viscosity (Jakobsen, Niss, and Olsen, 2005). These linear-response properties become frequency dependent 


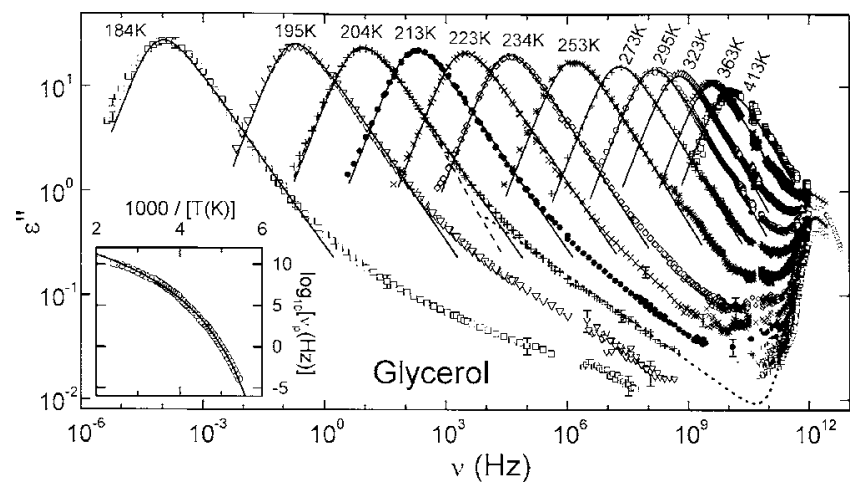

FIG. 3. Dielectric loss as a function of frequency for glycerol. The Debye dielectric function gives symmetric loss peaks in a log-log plot, but the data show the characteristic asymmetry towards higher frequencies that is almost always observed. Annealing for a long time is necessary at the lowest temperature to ensure that the liquid is in equilibrium. Inset: The loss peak frequency is non-Arrhenius. Reprinted from Schneider et al., 1998. Copyright 1998, with permission from Elsevier.

in viscous liquids at much lower frequencies than in other contexts of condensed-matter physics. Thus if $\tau$ $=1 \mathrm{~s}$, there is typically a nontrivial frequency dependence around $1 \mathrm{~Hz}$. Finally, computer simulations are increasingly used, although computers are still much too slow to simulate, e.g., a liquid with $\tau=1 \mathrm{~s}$.

When a viscous liquid is subjected to a sudden constant thermal, mechanical, or electrical perturbation, there is a slow relaxation towards steady state. This relaxation is almost always nonexponential (Mazurin, 1977; Scherer, 1990)-the first non. It is important to distinguish between linear and nonlinear relaxations, although both are usually nonexponential. ${ }^{3}$ In the linear case the perturbation in principle is infinitesimal. Linear perturbations are often studied in the frequency domain where a periodic input results in a periodic output. The ratio between output and input defines the relevant, complex linear-response function. Linear-response theory is based on the fluctuation-dissipation theorem, which allows one to calculate the response function from equilibrium fluctuations. As an example, an exponential dipole equilibrium time-autocorrelation function $\propto \exp \left(-t / \tau_{0}\right)$ results in the so-called Debye dielectric function $\epsilon(\omega) \propto 1 /\left(1+i \omega \tau_{0}\right)$. This is seldom observed; instead one typically finds a dielectric loss [negative imaginary part of $\epsilon(\omega)]$ as in Fig. 3. For many liquids there are additional, minor Johari-Goldstein "beta" peaks at higher frequencies than the dominant loss peak frequency.

The second non, our focus here, is the non-Arrhenius temperature dependence of the Maxwell "alpha" relaxation time $\tau$ or viscosity $\eta$ [according to Eq. (2) these are

\footnotetext{
${ }^{3}$ Both are often fitted by the so-called "stretched exponential" function $\exp \left[-\left(t / \tau^{\prime}\right)^{\beta}\right]$, where $0<\beta<1$ (Phillips, 1996). The characteristic time $\tau^{\prime}$ is usually not much different from the Maxwell relaxation time.
}

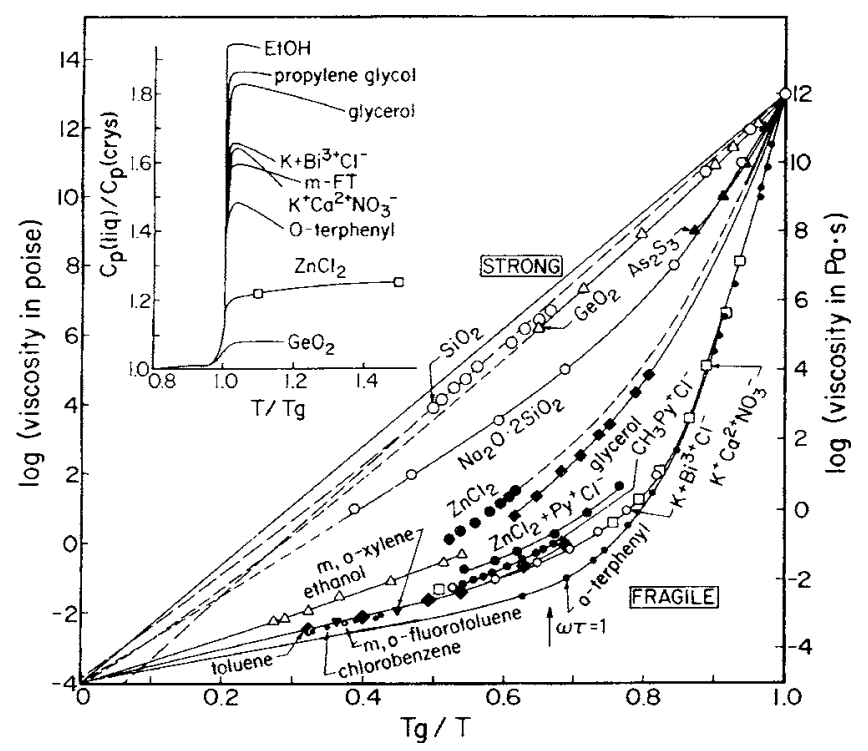

FIG. 4. The Angell (1985) fragility plot showing the viscosity as a function of inverse temperature normalized to 1 at $T_{g}$. The lower left corner marks the approximate high-temperature limit of the viscosity that is common to all liquids. An Arrhenius viscosity gives a straight line in this plot. Inset: Specific-heat jumps at the glass transition. Reprinted with permission.

roughly proportional since $G_{\infty}$ is much less temperature dependent than $\tau$ or $\eta$ ]. Figure 4 is the original "Angell plot" (1985) showing the logarithm of the viscosity for a number of viscous liquids as a function of inverse temperature normalized to unity at $T_{g}$, where $T_{g}$ is defined as the temperaure at which the equilibrium liquid viscosity is $10^{12} \mathrm{~Pa}$ s. If, as is generally assumed, dynamics are dominated by barriers to be overcome by thermal fluctuations, one would expect $\eta \sim \exp \left(\Delta E / k_{B} T\right)$ (Brush, $1962)$, corresponding to the diagonal line. This is referred to as an Arrhenius temperature dependence, after Arrhenius (1889) who discovered that chemical reaction times usually follow this law. The Arrhenius law, however, only works for a few liquids, e.g., pure silica $\left(\mathrm{SiO}_{2}\right)$ or phosphor pentoxide $\left(\mathrm{P}_{2} \mathrm{O}_{5}\right)$ (Varshneya, 1994). In the vast majority of cases viscous liquids show a stronger than Arrhenius increase of the viscosity upon cooling toward the glass transition. If one nevertheless accepts the Arrhenius expression, the activation energy must be temperature dependent: $\Delta E(T)$ is defined by ${ }^{4}$ (where $\tau_{0} \sim 10^{-13} \mathrm{~s}$ is a typical microscopic time)

$$
\tau=\tau_{0} \exp \left(\frac{\Delta E(T)}{k_{B} T}\right)
$$

The message of Fig. 4 is that the activation energy increases when temperature decreases. There seem to be no examples of viscous liquids with an activation energy that decreases upon cooling.

\footnotetext{
${ }^{4}$ More correctly, Eq. (4) defines the free energy of activation, but for simplicity we use the traditional term activation energy.
} 
For some viscous liquids $\tau$ increases by more than one order of magnitude when the temperature is lowered by just $1 \%$. Such strongly non-Arrhenius liquids are called "fragile" (no connection to glass fragility), while those closer to Arrhenius behavior are termed "strong" (Angell, 1985). Non-Arrhenius data are often fitted by the so-called Vogel-Fulcher-Tammann expression (Vogel, 1921; Fulcher, 1925; Tammann and Hesse, 1926)

$$
\tau=\tau_{0} \exp \left(\frac{A}{T-T_{0}}\right)
$$

Equation (5) implies that the liquid's equilibrium relaxation time becomes infinite at $T_{0}$, a prediction that cannot be verified because, if correct, the system is bound to fall out of equilibrium as $T_{0}$ is approached. Equation (5) has inspired leading theorists to speculate on its origin (Edwards, 1976; Anderson, 1979). Stillinger (1988) argued that a liquid cannot have an infinite relaxation time at a finite temperature. When compared to experiment, Eq. (5) generally breaks down in the highly viscous regime by predicting too large relaxation times (Brawer, 1985; Scherer, 1990; Angell, 1995; Stickel, Fischer, and Richert, 1995; Kivelson et al., 1996).

An alternative fit to data with the same number of parameters is provided by (Walther, 1931; Harrison, 1976; Bässler, 1987; Avramov, 2005):

$$
\tau=\tau_{0} \exp \left(\frac{C}{T^{n}}\right)
$$

No systematic analysis has investigated which of Eqs. (5) or (6) generally give the best fit to data.

The third non is the nonlinearity of relaxation for finite, but even quite small perturbations. For instance, upon a $2 \mathrm{~K}$ temperature jump to $206 \mathrm{~K}$ triphenyl phosphite initially relaxes more than one order of magnitude faster if starting at $208 \mathrm{~K}$ than starting at $204 \mathrm{~K}$. This lack of mirror symmetry is reproduced even by simple models (Bisquert, 2005). The nonlinearity derives from the fact that the relaxation time of an out-of-equilibrium liquid-a glass - depends not only on temperature, but also on the so-called fictive temperature (Scherer, 1986; Hodge, 1994; Avramov, 1996): Going from 208 to $206 \mathrm{~K}$ the fictive temperature starts at $208 \mathrm{~K}$ and ends up at $206 \mathrm{~K}$, which implies a faster relaxation than when the fictive temperature starts at $204 \mathrm{~K}$ to end up at $206 \mathrm{~K}$.

\footnotetext{
${ }^{5}$ This corresponds to the empirical classification into "short" and "long" liquids in glass industry, where short liquids are only amenable to processing for a brief period of time before solidifying as they cool. Silica-free commercial glass, for instance, is short and difficult to blow or draw (Rawson, 1988). The "working range" is the temperature between the "working point" (where a glass can be worked on, $\eta \sim 10^{3} \mathrm{~Pa} \mathrm{~s}$ ) and the "softening point" (where the worked mass will not deform under its own weight, $\eta \sim 10^{7} \mathrm{~Pa} \mathrm{~s}$ ) (Varshneya, 1994). The glass transition takes place much below the softening point.
}

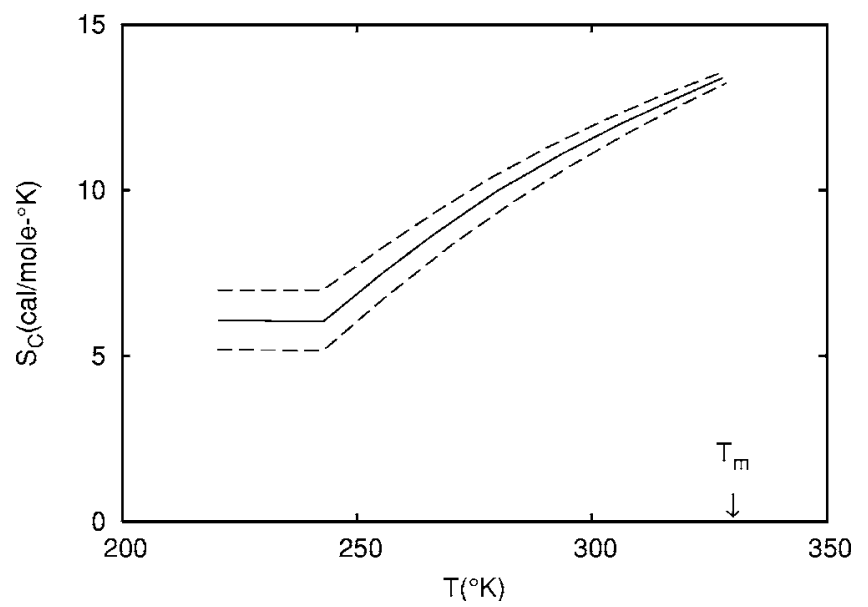

FIG. 5. Excess entropy (liquid minus crystalline) of supercooled ortho-terphenyl calculated from specific-heat data obtained at a cooling rate of $20 \mathrm{~K} / \mathrm{min}$ (the experimental uncertainty is marked by dashed lines). The excess entropy extrapolates to zero at a temperature above $200 \mathrm{~K}$, the Kauzmann temperature, but the glass transition intervenes just below $250 \mathrm{~K}$ and saves the situation. By slower cooling, however, it should be possible to take the equilibrium liquid to the Kauzmann temperature and below. This is the Kauzmann paradox. Reused with permission from Greet and Turnbull, 1967. Copyright 1967, American Institute of Physics.

\section{Kauzmann's paradox}

Just below the melting temperature the supercooled liquid has significantly larger entropy than the crystal. As the temperature is lowered, the liquid entropy usually drops much faster than the crystal entropy, however. Below a certain temperature $T_{K}$ termed the Kauzmann temperature, the liquid entropy by extrapolation becomes lower than the crystal entropy. But how can a liquid-which is disordered-have lower entropy than the same-temperature crystal? In real life the glass transition intervenes and saves the situation (Fig. 5), and $T_{K}$ is always identified by extrapolation. Since $T_{g}$ is lowered by slower cooling, however, it should be possible-at least in principle - to bring the liquid to equilibrium below $T_{K}$. This constitutes the Kauzmann paradox.

Simon (1930) first described this paradox for glycerol. Kauzmann (1948) discussed it in general terms and proposed the following solution: At deep supercooling the rate of homogeneous crystal nucleation becomes larger than $1 / \tau$, implying that no well-defined equilibrium liquid state exists (Cavagna, Attanasi, and Lorenzana, 2005). It is not clear why this would always happen before $T_{K}$ is reached, though, unless the relaxation time is bound to become infinite at $T_{K}$.

The specific heat is usually regarded as a sum of two contributions: a vibrational part which survives the glass transition, and a configurational part which is zero in the glassy phase (Goldstein, 1972). Similarly, the liquid entropy is a sum of two contributions. If, as traditionally assumed, the vibrational entropy of the liquid is close to that of the crystal, the configurational entropy $S_{\text {conf }}$ may 
be identified with the so-called excess entropy $S_{\text {exc }}$ $\equiv S_{\text {liq }}-S_{\text {cryst }}$. Thus $S_{\text {conf }}$ becomes zero at $T_{K}$, and it appears that something must happen here. One possibility is that there is a phase transition to a state of zero configurational entropy, an "ideal glass" (Gibbs and DiMarzio, 1958; Cohen and Turnbull, 1959; Angell, 1968; Anderson, 1979; Kirkpatrick, Thirumalai, and Wolynes, 1989). Another possibility is simply that the extrapolation is not valid (Stillinger, Debenedetti, and Truskett, 2001).

The idea of an underlying true phase transition is attractive. Even a simple two-level system, however, has an entropy which, if measured at high temperatures, extrapolates to zero at a finite temperature (Angell and Rao, 1972; Matyushov and Angell, 2005). In fact, liquid excess entropy data like those of Fig. 5 may be fitted by statistical mechanical models involving just a handful of energies (Goldstein, 1972). If the dynamics are described by a standard master equation, such simple models are even able to reproduce the hysteresis of the specific heat around the glass transition illustrated in Fig. 1(b) (Bisquert, 2005).

As mentioned, $S_{\text {conf }} \cong S_{\text {exc }}$ applies if the vibrational entropies of crystal and supercooled liquid are approximately the same. This was the prevailing opinion until recently, but it is now increasingly recognized that the assumption $S_{\text {config }} \cong S_{\text {exc }}$ most likely is incorrect (Goldstein, 1976; Phillips et al., 1989; Johari, 2002, 2003). In fact, there was always a good reason to be skeptical towards the identification of liquid and crystalline vibrational entropies: When the frequency is much larger than $1 / \tau$, the sound velocity reflects short-time scale

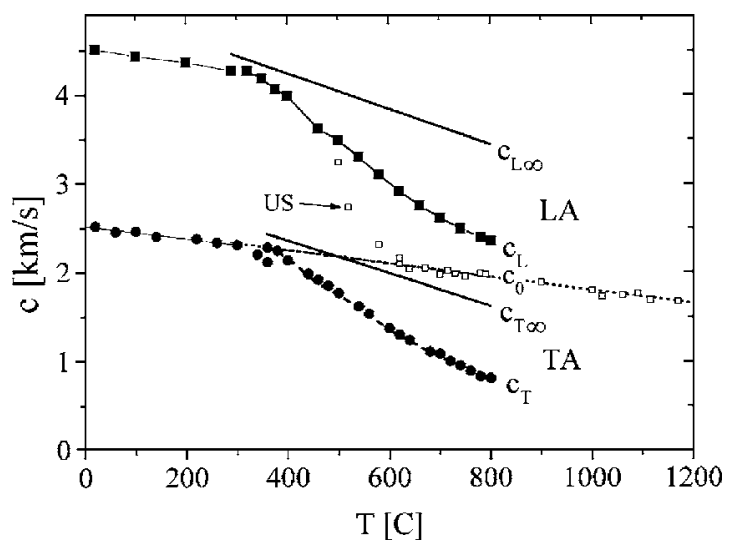

FIG. 6. High-frequency longitudinal (LA) and transverse (TA) sound velocities of the mainly ionic salt-melt mixture comprising $94 \% \mathrm{NaPO}_{3}$ and $6 \% \mathrm{Al}\left(\mathrm{PO}_{3}\right)_{3}$ with data obtained from Brillouin scattering both above and below the glass transition (around $350 \mathrm{~K}$ ). Full symbols give the apparent sound velocities estimated from peak positions; full lines give model-based high-frequency sound velocities. The high-frequency sound velocity is clearly more temperature dependent in the liquid phase than in the glass phase. This implies that the vibrational entropy of the liquid phase has a temperature dependence different from that of the crystalline phase. Adapted from Soltwisch et al., 1999, with permission from Taylor \& Francis Ltd. elastic properties where the liquid molecules just vibrate around fixed positions. Consider the data of Fig. 6, which are similar to high-frequency sound velocity measurements of glass-forming liquids obtained with ultrasonic methods in the 1950s and 1960s (Litovitz, 1959; Barton, 1972). The smaller the high-frequency sound velocity $c_{\infty}$ is, the larger the vibrational entropy is $\left[S_{\text {vib }}\right.$ $\propto \ln \left(1 / c_{\infty}\right)$-more on this later]. Figure 6 shows that the high-frequency sound velocity is more temperature dependent in the liquid phase than in the glass phase. Usually the crystal and glass are similar as regards the temperature variation of the sound velocities. Therefore the vibrational entropies of the crystal and supercooled liquid cannot in general be approximately the same.

How serious is the Kauzmann paradox? In view of the above it appears that there may be no fundamental problem if the entropy of an equilibrium supercooled liquid at some temperature is lower than that of the corresponding crystal. An example of this is "inverse melting," where a liquid crystallizes upon heating and the liquid phase consequently has lower entropy than the crystalline phase (Mortensen, Brown, and Nordén, 1992; Stillinger and Debenedetti, 2003).

\section{MODELS OF THE CONVENTIONAL WISDOM}

All molecules of a viscous liquid have the thermal velocities prescribed by the canonical ensemble, but virtually all motion goes into vibrations. Thus it has been suggested that a viscous liquid is to be regarded more as "a solid that flows" than as a conventional liquid (Dyre, 1999, 2005, 2006). This is consistent with the longstanding assumption that viscous-liquid thermodynamics may be separated into a vibrational and a configurational part. The dynamics also separate into vibrations on the short time scale and "inherent dynamics," i.e., jumps between potential energy minima on the long time scale (Goldstein, 1969; Stillinger and Weber, 1983; Schrøder et al., 2000; Sciortino, 2005).

An obvious question is: Given that silicates, alcohols, molten salts, aqueous solutions, and metallic melts have little in common chemically, is there any reason to expect that a general theory exists for the dynamics of glass-forming liquids? One reason to be optimistic is the following. As a general rule in physics, whenever a phenomenon is characterized by a small dimensionless number, there is a good chance that a simple description exists. ${ }^{6}$ If $D$ is the molecular diffusion constant and $\nu$ $=\eta / \rho$ is the so-called dynamic viscosity $(\rho$ is the density), we define $\gamma \equiv D / \nu$. In the hightemperature, less-viscous phase one has $\gamma \sim 1$ (Boon and Yip, 1980; Hansen and McDonald, 1986). Upon cooling

\footnotetext{
${ }^{6} \mathrm{~A}$ well-known example is the ideal gas law which applies to a good approximation whenever $r / \lambda \ll 1$, where $r$ is the molecule size and $\lambda$ the mean-free path.
} 
$D$ decreases roughly as $1 / \eta{ }^{7}$ while at the same time $\nu$ increases proportionally to $\eta$. At the glass transition one finds $\gamma \sim 10^{-30}$, which is an unusually small number in condensed-matter physics.

From here on we focus on the second non: Why are glass-forming liquids non-Arrhenius? The traditional measure of non-Arrhenius behavior is the "fragility" $m$ defined (Plazek and Ngai, 1991; Böhmer et al., 1993; Ruocco et al., 2004) by

$$
\left.m \equiv \frac{d \log _{10} \tau}{d\left(T_{g} / T\right)}\right|_{T_{g}} .
$$

If the relaxation time prefactor of Eq. (4) is $10^{-13} \mathrm{~s}$ and the glass transition temperature is defined by $\tau\left(T_{g}\right)$ $=10^{3} \mathrm{~s},{ }^{8}$ Arrhenius behavior is characterized by $m=16$. Only a few glass formers have fragility below 25 . Glycerol is intermediate with $m \simeq 50$, while, e.g., the molten salt $\mathrm{K}_{3} \mathrm{Ca}_{2}\left(\mathrm{NO}_{3}\right)_{7}$ has $m \simeq 90$. A high-fragility liquid is decalin $(m \simeq 150)$.

The stronger than Arrhenius behavior derives from $\Delta E$ increasing with decreasing temperature. An alternative measure of the degree of non-Arrhenius behavior is provided by the "index" $I=I(T)$ (Schug, King, and Böhmer, 1998; Granato, 1999, 2002; Dyre and Olsen, 2004):

$$
I \equiv-\frac{d \ln \Delta E}{d \ln T} .
$$

$I$ quantifies Arrhenius deviations in a way inspired by the Grüneisen parameter (Kittel, 1996). A straightforward calculation shows that the fragility is related to the index by

$$
m=16\left[1+I\left(T_{g}\right)\right] .
$$

The Arrhenius case has $I=0$ and $m=16$. If $\tau(T)$ is given by Eq. (6), the index is temperature independent: $I=n$ -1 . Typical indices of glass-forming liquids range from 2 to 7. A theory of viscous liquid dynamics must explain why the activation energy has such a relatively strong temperature dependence.

\section{A. Entropy model}

The idea here is that the long relaxation times at low temperatures are brought about by a dearth of configurations (Gibbs and DiMarzio, 1958; Adam and Gibbs, 1965). Assuming that molecular reorientations take place cooperatively, Adam and Gibbs (1965) argued that the minimum size of a cooperatively rearranging region

\footnotetext{
${ }^{7}$ According to the Stokes-Einstein relation the molecular diffusion constant is proportional to the inverse viscosity, reflecting the fact that high viscosity impedes self-motion. The Stokes-Einstein relation is often violated in viscous liquids by 1-3 orders of magnitude (Diezemann et al., 1998; Douglas and Leporini, 1998), but this is not important for the above argument.

${ }^{8}$ Since $G_{\infty} \sim 10^{9}-10^{10} \mathrm{~Pa}$ this approximately corresponds to defining $T_{g}$ by $\eta\left(T_{g}\right)=10^{12} \mathrm{~Pa} \mathrm{~s}$ [compare Eq. (2)].
}

is determined by the requirement that it should contain at least two different configurational states. As the temperature is lowered the cooperatively rearranging regions grow. Assuming that the activation energy is proportional to the region volume, the model predicts that $\tau=\tau_{0} \exp \left[C / T S_{\text {conf }}(T)\right]$ which implies a stronger than Arrhenius behavior. If the identification $S_{\text {config }} \cong S_{\text {exc }}$ is accepted and the Kauzmann paradox is formulated as $S_{\text {conf }}(T) \propto T-T_{K}$, the entropy model to leading order implies Eq. (5) with $T_{0}=T_{K}$ (Angell, 1997).

The entropy model connects dynamics to thermodynamics by assuming that the often dramatic increase of the relaxation time upon cooling reflects the existence of an underlying second-order phase transition to a state of zero configurational entropy, a state usually termed an ideal glass. This is an attractive scenario, especially to the generation of theorists who grew up with the theory of critical phenomena of the 1970s and 1980s (Wilson, 1983; Goldenfeld, 1992). For several years the entropy model has dominated the field to the extent that it defined a paradigm. There are, however, a number of problems with this model: (1) On the conceptual level one might ask what the ideal glass state is. Since it is unique, a simple description of it would be expected, but none has been proposed. (2) A further conceptual problem is that model parameters typically imply cooperatively rearranging regions containing just four to eight molecules at the glass transition and even fewer at higher temperatures (Yamamuro et al., 1998). This is not enough to justify the assumption of cooperatively rearranging regions acting independently of their surroundings. (3) The assumption that the energy barrier is proportional to the region volume is ad hoc. (4) The entropy model's experimental validation is based on assuming $S_{\text {conf }} \cong S_{\text {exc }}$ which as mentioned is problematic. If the configurational entropy is a temperature-independent fraction of the excess entropy (Martinez and Angell, 2001; Prevosto et al., 2003; Corezzi, Comez, and Fioretto, 2004), the model nevertheless predicts $\tau \propto \exp \left(C / T S_{\text {exc }}\right)$, but it is not obvious why this should be the case (Johari, 2002, 2003). (5) A recent compilation of data led to the conclusion that the identity $T_{0} \cong T_{K}$, previously believed to give a good description of the overall experimental situation, does not apply in general (Tanaka, 2003).

\section{B. Free-volume models}

The basic idea here is that molecules need "free" volume in order to be able to rearrange. As the liquid contracts upon cooling, less free volume becomes available. If the free volume per molecule is denoted by $v_{f}$, the model prediction is $\tau=\tau_{0} \exp \left(C / v_{f}\right)$ (Cohen and Turnbull, 1959; Grest and Cohen, 1981). There are several mutually inconsistent free-volume models (Kovacs, 1963) - the problem is that it is not possible to define free volume rigorously. Cohen and collaborators defined it as that part of the volume "which can be redistributed without energy cost" and argued that this quantity goes to zero at a finite temperature. This leads to Eq. (5) if 
$v_{f}(T)$ is expanded to first order. Doolittle (1951) defined the free volume by subtracting the molecular volume defined by extrapolating the liquid volume to zero temperature, implying that $v_{f} \rightarrow 0$ only when $T \rightarrow 0$.

Free-volume models are not generally popular because the relaxation time is not just a function of density $\rho$. Recent comprehensive experiments, which varied both temperature and pressure, may be summarized into the scaling law $\Delta E / k_{B} T=F\left(\rho^{x} / T\right)$ where $x$ and the function $F$ are both nonuniversal (Alba-Simionesco et al., 2004; Casalini and Roland, 2004).

\section{Other models}

The entropy model and free-volume models link the activation energy to a macroscopic observable. Besides entropy and volume, a third class of models of this kind takes energy as the controlling variable (Goldstein, 1972; Nemilov, 1978; Brawer, 1984, 1985; Bässler 1987; Dyre, 1987; Bouchaud, 1992; Diezemann, 1997). In the simplest version it is assumed that thermal excitation takes place to a specific barrier energy $E_{0}$ (Goldstein, 1972; Brawer, 1984; Dyre, 1987; Bouchaud, 1992). If the process is cooperative, the most likely energy of a rearranging region is close to its average energy $\bar{E}(T)$. This implies that $\Delta E(T) \cong E_{0}-\bar{E}(T)$, so as temperature is lowered, the activation energy increases. This model implies too broad linear-response loss peaks, however, unless the cooperatively rearranging regions are quite small (Dyre, 1995). Thus if energy is the variable controlling $\tau$, a different route must be taken (Diezemann, 2005).

A first-principles approach is provided by the modecoupling theory that starts with Newton's equations of motion and after several nontrivial approximations ends up with definite experimental predictions (Götze and Sjögren, 1992; Sokolov, 1997; Das, 2004; Kob, 2004). In ideal mode-coupling theory the dynamics are determined by static equilibrium averages, a most attractive feature. The theory predicts a critical temperature below which there is no ergodic phase. There are extensions of mode-coupling theory which by taking activated processes into account broaden the theory's range of applicability to lower temperatures. Nevertheless, while mode-coupling theory is believed to correctly explain the onset of viscous behavior upon cooling, the consensus is that it breaks down well before the glass transition is approached (Dreyfus and Pick, 2001).

Many other ways to understand the non-Arrhenius property of glass-forming liquids have been proposed, e.g., the energy landscape approach (Goldstein, 1969; Stillinger, 1995; Debenedetti and Stillinger, 2001; Wales, 2003; Sciortino, 2005); models with trivial thermodynamics, but nontrivial dynamics (Fredrickson, 1988; Pitts, Young, and Andersen, 2000; Garrahan and Chandler, 2002; Ritort and Sollich, 2003; Pedersen et al., 2006); the random first-order transition theory (Kirkpatrick, Thirumalai, and Wolynes, 1989; Xia and Wolynes, 2000); the entropic barrier hopping theory (Schweizer and Saltz- man, 2004); and the theory of frustration-limited domains (Tarjus et al., 2005).

Most models of the conventional wisdom predict that the viscous slowing down is a consequence of an underlying or narrowly avoided phase transition (Sethna, 1988; Kivelson et al., 1995). Looking at data like those of Fig. 4, however, one might ask with Occam's razor in mind: Is the assumption of an underlying phase transition really compelling?

\section{ELASTIC MODELS: CONNECTING THE FAST AND SLOW DEGREES OF FREEDOM}

If $V$ is volume and $p$ pressure, the (adiabatic/ isothermal) bulk modulus $K$ is defined by $K$ $\equiv-V(\partial p / \partial V)=-\partial p / \partial(\ln V)$. For systems with positive thermal expansion the adiabatic bulk modulus is higher than the isothermal because there is heating associated with an adiabatic compression. The adiabatic and isothermal shear moduli, on the other hand, are always identical.

Any liquid is solidlike when probed on a sufficiently short time scale; its short-time elastic properties are characterized by the instantaneous moduli $G_{\infty}$ and $K_{\infty}$. When the relaxation time is, e.g., one second or longer, it may be difficult to imagine that relaxation processes can have any relation to the elastic properties on the picosecond time scale. Surely, one cannot predict the rate of global climate variations over millions of years from observations of the world's weather collected over a single minute. Nevertheless, the barrier transition for a "flow event" (a molecular rearrangement) does take place on a very short time scale, so the height of the energy barrier could well be determined by liquid properties which can be probed on this time scale. The elastic models all embody this idea one way or the other.

\section{A. Harmonic approximation}

The first paper suggesting that the activation energy is determined by short-time elastic properties appears to be a little known work by Tobolsky, Powell, and Eyring (1943) basically arguing as follows. The viscosity is determined by the rate of molecules moving from one equilibrium position (energy minimum) to another. In the schematic situations of Fig. 7 the energy barrier to be overcome is clearly overestimated by the intersection of the parabolic extrapolations from the minima. Comparing the situations of Figs. 7(a) and 7(b), however, the barrier is overestimated by the same numerical factor. Thus the estimated barrier is proportional to the actual barrier ( $a$ only changes insignificantly). According to rate theory (Kramers, 1940) the average time between jumps $\tau$ is a microscopic time $\tau_{0}$ divided by the statistical mechanical probability to find the system around the energy maximum. In the harmonic approximation the energy is quadratic in the "reaction coordinate"; this leads to a Gaussian statistical-mechanical probability distribution $\propto \exp \left(-x^{2} / 2\left\langle x^{2}\right\rangle\right)$, where $x$ is the reaction coordinate 


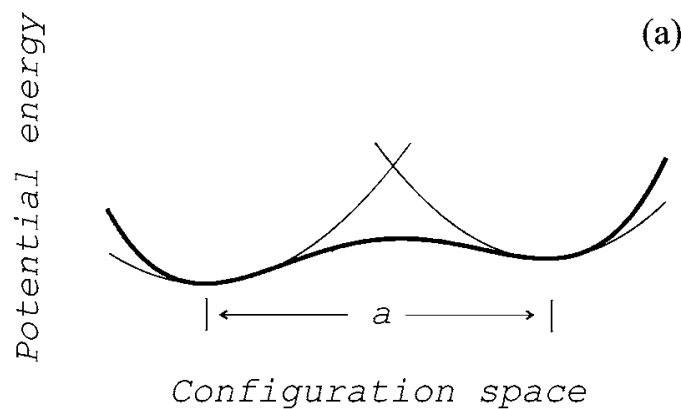

(b)

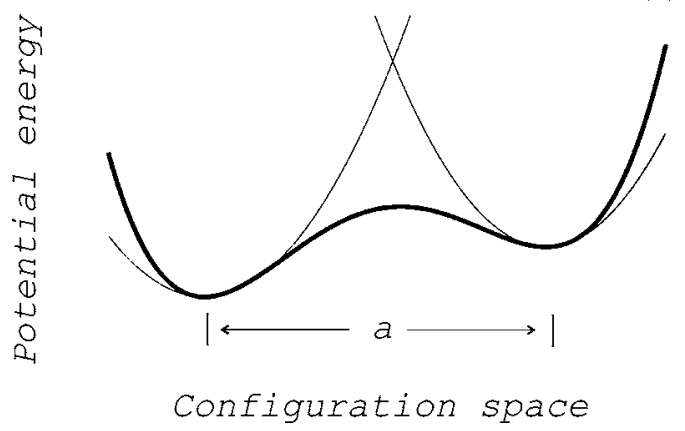

FIG. 7. Comparing two cases of potential energy minima with different curvatures at the minima. The full curve is the potential energy; the thin curve gives the potential estimated by second-order Taylor expansions around the minima. The barriers estimated from the intersection of thin curves are considerably larger than the actual barriers; however, going from (a) to (b) the estimated and actual barriers are proportional.

deviation from its value at the minimum and $\left\langle x^{2}\right\rangle$ is the vibrational mean-square thermal average around one minimum. Thus for some numerical factor of order one $\lambda_{1}$, if $a$ is the average intermolecular distance, the harmonic approximation leads to

$$
\tau=\tau_{0} \exp \left(\lambda_{1} \frac{a^{2}}{\left\langle x^{2}\right\rangle}\right) .
$$

Comparing to Eq. (4) the activation energy is given by

$$
\Delta E=\lambda_{1} k_{B} T \frac{a^{2}}{\left\langle x^{2}\right\rangle} .
$$

The vibrational mean-square displacement $\left\langle x^{2}\right\rangle$ depends on temperature. For a harmonic, temperatureindependent system the intermolecular potential is parabolic and classical equipartition implies that $\left\langle x^{2}\right\rangle \propto T$, so $\Delta E$ is temperature independent. For most glass-forming liquids $\left\langle x^{2}\right\rangle$ decreases faster than $T$ upon cooling, resulting in an activation energy that increases upon cooling. During the last 20 years Eq. (10) or related expressions were derived and discussed by a number of authors in different contexts of viscous liquid dynamics (Hall and Wolynes, 1987; Buchenau and Zorn, 1992; Sokolov et al., 1994; Novikov et al., 1996; Roland and Ngai, 1996; Teeter et al., 2001; Starr et al., 2002; Bordat et al., 2004; Ngai,
2004). Equation (10) has also been used for diffusion in crystals (Flynn, 1968; Köhler and Herzig, 1988).

Returning to the 1943 paper of Eyring and collaborators, these authors further reasoned that the relevant potential is that resisting shear deformation, implying that $\Delta E \propto G$ where $G$ is the "liquid shear modulus." A liquid has zero dc shear modulus, and clearly the authors referred to the instantaneous shear modulus, leading to the following expression (where $\lambda_{2} \sim 1$ and the microscopic volume $a^{3}$ comes from a dimensional analysis):

$$
\Delta E=\lambda_{2} a^{3} G_{\infty} .
$$

It has been known for many years that $G_{\infty}$ is usually much more temperature dependent in viscous liquids than in crystals, glasses, or less-viscous liquids, and that $G_{\infty}$ always increases when temperature is lowered (Barlow et al., 1967; Harrison, 1976; Lamb, 1978).

\section{B. Models of Mooney and Bueche}

Mooney (1957) assumed that a local molecular rearrangement, a "quantum of liquid flow," comes about when thermal fluctuations generate a local expansion exceeding a certain critical value. He estimated how frequently such an expansion occurs by calculating the probability that thermal longitudinal sound waves by chance interfere to create a critically expanded local volume. If $c_{\infty}$ is the high-frequency longitudinal sound velocity and $m$ the molecular mass (not to be confused with the fragility), Mooney's calculation results in (where $\left.\lambda_{3} \sim 1\right)$

$$
\Delta E=\lambda_{3} m c_{\infty}^{2} \text {. }
$$

Bueche (1959) considered viscous liquid flow along similar lines, arguing as follows. A molecule is regarded as surrounded by a spherical layer of nearest neighbors, which is surrounded by a second shell of molecules, and so on. The shell radii fluctuate thermally, but the shells are assumed to stay spherical. "If all these concentric shells should vibrate outward in phase, then the innermost shell would expand greatly. This would leave the central molecule in a rather large hole and it would move into a new position." Bueche's calculation of the probability of this happening is less reliable than Mooney's, but the physical pictures are similar in two crucial assumptions: (1) The free-volume-like assumption that flow events only take place when there is a local, temporary density decrease. (2) The probability of this happening is determined by the short-time elastic properties of the liquid.

\section{Nemilov's approach}

Nemilov $(1968,1992,1995)$ derived Eq. (12) by substituting expressions for the viscosity and relaxation time into the Maxwell relation Eq. (2). He used Eyring's (1936) expression for the viscosity: $\eta=\left(h / a^{3}\right)$ $\times \exp \left(\Delta E / k_{B} T\right)$, where $h$ is Planck's constant. For the relaxation time he used the Dushman (1920) theory of 


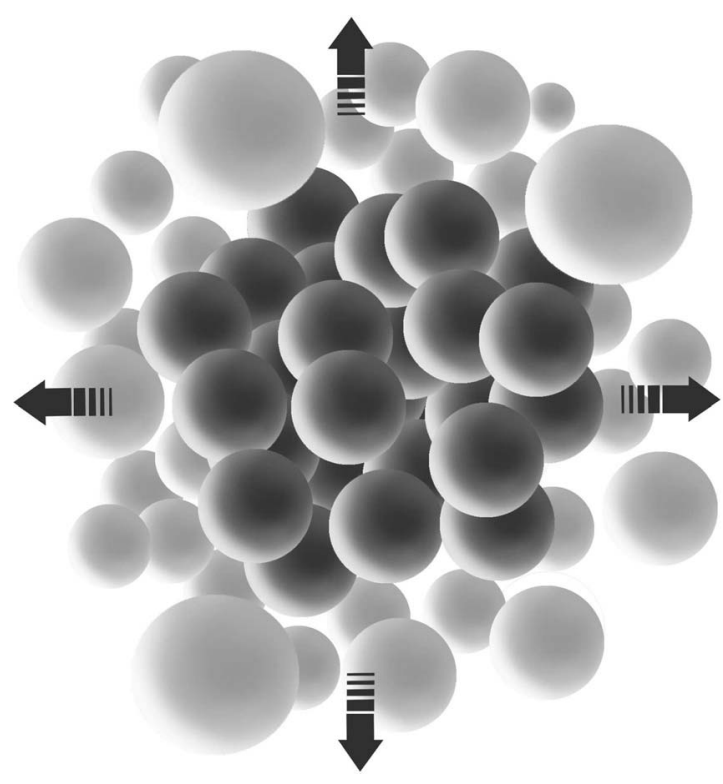

FIG. 8. A flow event (a molecular rearrangement) according to the shoving model with the dark spheres showing molecules before the flow event. A flow event takes place if the region volume briefly expands sufficiently due to a thermal fluctuation. According to statistical mechanics the probability of this happening is the reversible work done on the surrounding fixed structure to bring about the same expansion, a quantity that is calculated using standard solid-state elasticity theory. In the simplest case, that of spherical symmetry, the surroundings are subjected to a pure shear deformation and the activation energy is proportional to $G_{\infty}(T)$.

unimolecular reaction velocities with $\tau$ identified with the inverse reaction rate: $\tau=(h / \Delta E) \exp \left(\Delta E / k_{B} T\right)$. The result is Eq. (12) with $\lambda_{2}=1$.

\section{Shoving model}

Many liquid properties derive simply from the fact that the intermolecular forces consist of short-ranged, harsh repulsions and long-ranged, much weaker attractions (Widom, 1967; Chandler, Weeks, and Andersen, 1983). Because of the harsh repulsion it is reasonable to assume that, unless extra room is temporarily created, a molecular rearrangement requires very high energy. The shoving model assumes that the activation energy is dominated by the work done to shove aside the surroundings (Dyre, Olsen, and Christensen, 1996).

As a simple model (Fig. 8), suppose that the region of rearranging molecules is approximately spherical and that the radius must increase by $\Delta r$ before a flow event has a fair chance of taking place. Quite generally, the probability of a thermal fluctuation is the frequency (i.e., how often the fluctuation happens per unit time) times the average time spent at the fluctuating state. Since the time spent close to the barrier maximum is independent of barrier height, the frequency is proportional to the probability of finding the system around the barrier maximum.
According to statistical mechanics the probability of a thermal fluctuation resulting in a local density decrease is $\exp \left(-W / k_{B} T\right)$, where $W$ is the reversible work done on the system by external forces to bring about the required local expansion. To calculate $W$ in the fixed structure of liquid molecules vibrating around a given potential-energy minimum, the system is regarded as a solid with elastic constants $G_{\infty}$ and $K_{\infty}$. W is calculated as reversible work by assuming mechanical equilibrium, and the displacements in the surroundings are found using standard linear-elasticity theory. Calculating the work done to enlarge a sphere in an isotropic solid is a classical exercise of elasticity theory (Landau and Lifshitz, 1970). The result is that, if $r$ is the distance to the center, the (radial) displacement varies as $r^{-2}$. This is a pure shear displacement, i.e., not associated with any density changes. What happens in the surroundings of a flow event is the analog of Hilbert's hotel, the infinite hotel that even when totally occupied can always host an extra guest (simply by asking each guest to move to one higher room number).

Since the displacement field induces no density changes in the surroundings, the relevant elastic constant is $G_{\infty}$. The work is proportional to $G_{\infty}$ because the force is, thus leading to Eq. (12). The shoving model philosophy differs from that of Mooney (1957), who argued that "concentrating free volume in one region happens only at the expense of producing higher density in other regions."

To summarize, the three assumptions of the shoving model are

- the activation energy is mainly elastic energy;

- this elastic energy is mainly located in the surroundings of the flow event;

- the elastic energy is mainly shear elastic energy.

How realistic are these assumptions? That the energy barrier is mainly elastic energy associated with relatively small displacements of the surrounding molecules may be justified as follows (Dyre, 1998). The energy cost associated with the rearranging molecules themselves is a function of the expansion of their sphere $f(\Delta r)$. Because of the harsh repulsions, $f(\Delta r)$ is expected to decrease considerably if $\Delta r$ is just slightly increased from zero. Mathematically, this translates into $|d \ln f / d \ln \Delta r| \gg 1$. The "shoving" work on the surroundings is a quadratic function $A(\Delta r)^{2} / 2$. Minimizing the sum of these two terms in order to identify the optimal $\Delta r$ leads to $A \Delta r$ $+f^{\prime}(\Delta r)=0$. The ratio between the shoving work and the inner energy cost is $A(\Delta r)^{2} / 2 f(\Delta r)=|d \ln f / d \ln \Delta r| / 2 \gg 1$; thus the shoving work gives the dominant contribution to the activation energy.

\section{E. How do elastic models compare to experiment?}

Elastic models have been compared to experiment in a number of ways, but more work is needed before a conclusion can be reached regarding their overall ability to describe the non-Arrhenius relaxation time. Nemilov 


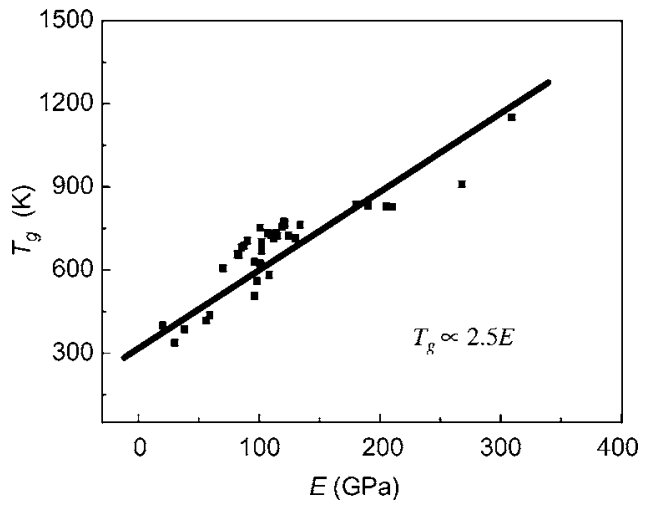

FIG. 9. Young's modulus $E$ vs the glass transition temperature for metallic glasses for which data are presently available. There is a linear relation between these two quantities as predicted by elastic models, although the line does not go through the origin as required to give a perfect model fit. Similar plots with $E$ replaced by $G$ were presented by Nemilov (1992) for other classes of glass-forming liquids. Reprinted from Wang, 2005. Copyright 2005, with permission from Elsevier.

(1992, 1995) compared Eq. (12) to experiment by arguing as follows. Since the structure freezes at $T_{g}$, the glass shear modulus (which varies only a little with temperature) is approximately $G_{\infty}\left(T_{g}\right)$. The glass transition takes place when $\tau \sim 10^{3} \mathrm{~s}$ and the prefactor of Eq. (4) is given by $\tau_{0} \sim 10^{-13} \mathrm{~s}$, so the ratio $\Delta E / k_{B} T_{g}$ is a universal constant at $T_{g}$. Thus for chemically closely related glasses, i.e., with same intermolecular distance $a$, Eq. (12) predicts that $T_{g}$ is proportional to the glass shear modulus $G$ (Sanditov, Sangadiev, and Kozlov, 1998). For each of four types of glasses (silica-based optical glasses, germanate glasses, $\mathrm{BeF}_{2}$-based glasses, and chalcogenide glasses) Nemilov (1992) found a linear relationship between $T_{g}$ and $G$, though not in all cases an exact proportionality. Figure 9 shows recent data along similar lines for bulk metallic glasses (Wang, 2005, 2006), showing a linear relation between $T_{g}$ and Young's modulus $E$ [defined by $1 / E=1 /(3 G)+1 /(9 K)$ (Landau and Lifshitz, 1970)]. A related line of reasoning notes that if $\lambda_{3}$ of Eq. (13) is a universal constant, $T_{g}$ should be proportional to $m c_{\infty}^{2}\left(T_{g}\right)$. Heuer and Spiess (1994) showed that this applies to a good approximation where $c_{\infty}\left(T_{g}\right)$ was identified with the glass sound velocity.

When Eq. (11) is compared to experiment it is common to subtract the crystalline vibrational mean-square displacement extrapolated into the liqiuid regime. After this procedure the equation is able to describe a number of data (Buchenau and Zorn, 1992; Kanaya et al., 1999; Magazu et al., 2004; Cornicchi, Onori, and Paciaroni, 2005). If this is general, it appears that Eq. (11) somewhat underestimates the degree of non-Arrhenius behavior.

The shoving model was compared to data on molecular liquids obtained with the piezo-ceramic method (Christensen and Olsen, 1995) optimized for measuring high shear moduli of viscous liquids approaching $T_{g}$ (Dyre, Olsen, and Christensen, 1996; Jakobsen, Niss,

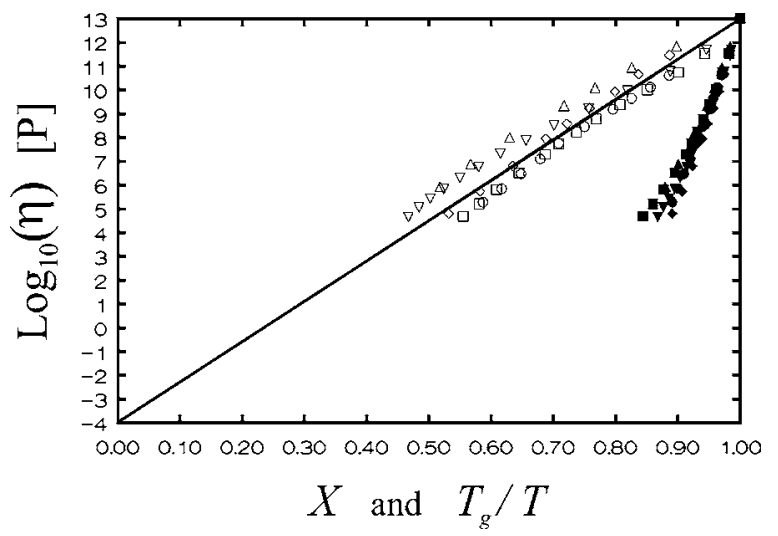

FIG. 10. Viscosity as a function of inverse temperature (full symbols) and as function of $X \propto G_{\infty} / T$ (open symbols) for four organic liquids and one silicone oil with both $x$-axis variables normalized to 1 at $T_{g}(1 \mathrm{P}=0.1 \mathrm{~Pa} \mathrm{~s})$. Results were obtained with a piezo-ceramic measuring method (Christensen and Olsen, 1995). As in Fig. 4, the approximate high-temperature limit of the viscosity is given at the lower left corner. Open symbols follow the diagonal line predicted by the shoving model ending in the lower-left corner at a typical hightemperature viscosity. Adapted from Dyre, Olsen, and Christensen, 1996.

and Olsen, 2005). This method makes it possible to measure the dc viscosity and the frequency-dependent shear modulus over 7-8 decades up to $50 \mathrm{kHz}$. The results (Fig. 10) show that $\log \eta$ is a linear function of $G_{\circ}(T) / T$ with a physically reasonable prefactor, as predicted by Eq. (12). It is not obvious, however, that frequencies in the $100 \mathrm{kHz}$ range are high enough to ensure that $G_{\infty}$ is measured. Transverse Brillouin light scattering gives data in the $\mathrm{GHz}$ range, but even these data may be affected by possible relaxation processes. Here one finds that the log-log temperature derivative of $G_{\infty}$ [activation energy index, Eq. (8)] is often (but not always) somewhat smaller than required to fit experiment.

\section{COMMON FEATURES OF ELASTIC MODELS}

\section{A. Equivalence of elastic models in the simplest approximation}

The fluid sound velocity is given by the well-known expression $c^{2}=\partial p / \partial \rho$, where the derivative exists in adiabatic and isothermal versions. In most cases the adiabatic version applies because the system does not have time to thermalize locally when a sound wave passes by. In terms of the adiabatic bulk modulus $K=-\partial p / \partial(\ln V)$ $=\partial p / \partial(\ln \rho)$ the sound velocity is given by $\rho c^{2}=K$.

Sound waves in ordinary fluids are longitudinal, i.e., with displacement in the direction of the propagating wave. In solids there are also transverse (shear) waves, waves that do not result in density changes. The sound velocity of a shear wave is given by $\rho c^{2}=G$. The sound velocity of a longitudinal wave in a solid is given by $\rho c^{2}=K+4 G / 3$, which is a generalization of the fluid $(G$ $=0$ ) case (Harrison, 1976). 
The fluid "identity" $G=0$ applies only at frequencies much below $1 / \tau$. For highly viscous liquids this leaves a broad range of frequencies with a significant nonzero shear modulus, and at high frequencies $(\omega \tau \gg 1)$ the sound velocities are given by the solid expressions. Thus if " $t$ " and " $l$ " refer to transverse and longitudinal sound waves, respectively, and $K_{\infty}$ is the high-frequency adiabatic bulk modulus, the high-frequency sound velocities are given by

$$
\begin{aligned}
& \rho c_{t, \infty}^{2}=G_{\infty}, \\
& \rho c_{l, \infty}^{2}=K_{\infty}+\frac{4}{3} G_{\infty} .
\end{aligned}
$$

Since $\rho=m / a^{3}$ by definition of $a$, Eq. (14) implies that Eqs. (12) and (13) are equivalent if the instantaneous shear and bulk moduli are proportional in their temperature variations.

A connection to Eq. (11) is established by noting that for a one-dimensional potential $U(x)=U_{0}+(1 / 2) m \omega_{0}^{2} x^{2}$ the classical equipartition theorem, $\langle U\rangle=U_{0}+(1 / 2) k_{B} T$, implies that $m \omega_{0}^{2}\left\langle x^{2}\right\rangle=k_{B} T$. Generally, if vibrations are modeled harmonically by having a single force constant written as $m \omega_{0}^{2}$, one finds the same result by expanding on a complete set of waves, thus $a^{2} /\left\langle x^{2}\right\rangle \propto m a^{2} \omega_{0}^{2} / k_{B} T$. In these relations the characteristic frequency $\omega_{0}$ is effective and may depend on temperature. In this "singleforce-constant model" the transverse and longitudinal sound velocities are proportional; by dimensional arguments one concludes that both are given by $c_{\infty} \propto a \omega_{0}$. This implies that $a^{2} /\left\langle x^{2}\right\rangle \propto m c_{\infty}^{2} / k_{B} T$. To summarize, in the single-force-constant model Eqs. (11)-(13) are equivalent in their temperature variations:

$$
\frac{\Delta E}{k_{B} T} \propto \frac{m c_{\infty}^{2}}{k_{B} T} \propto \frac{a^{3} G_{\infty}}{k_{B} T} \propto \frac{a^{2}}{\left\langle x^{2}\right\rangle} .
$$

If the proportionality constants are universal, Eq. (15) implies a Lindemann criterion for the glass transition: Recall that the Lindemann (1910) criterion is the rule that melting of a crystal takes place when the average vibrational displacement exceeds $10 \%$ of the nearestneighbor distance. As noted already, at the glass transition the activation energy relative to $T_{g}$ is a universal number. Thus Eq. (15) implies that the ratio $a^{2} /\left\langle x^{2}\right\rangle$ is universal at $T_{g}$, which is a Lindemann criterion for the glass transition.

The glass transition Lindemann criterion is supported by data: When glasses are prepared by standard slow cooling, glass moduli usually do not differ much from those of the crystal. This means that if the classical harmonic approximation $\left\langle x^{2}\right\rangle \propto T$ applies in both the crystalline and glassy phases, the proportionality constant is roughly the same for both phases. Thus the Lindemann criterion implies that $T_{g}$ is a definite fraction of the melting temperature $T_{m}$. This indeed applies, since $T_{g} / T_{m}$ in the vast majority of cases varies between 0.5 and 0.8 . In particular, the glass transition Lindemann criterion explains why one never observes $T_{g} \ll T_{m}$.
Elastic models offer a simple scenario for explaining the glass transition Lindemann criterion, but this criterion was also arrived at from arguments based on excess low-energy vibrational states in glasses (Malinovsky and Novikov, 1992), the existence of a single energy scale (Heuer and Spiess, 1994), percolation theory (Novikov et al., 1996), energy landscape arguments (Onuchic, Luthey-Schulten, and Wolynes, 1997), free-volume theory (Sanditov and Sangadiev, 1998; Starr et al., 2002; Berry and Smirnov, 2005), random first-order transition theory (Xia and Wolynes, 2000), computer simulations (Starr et al., 2002; Bordat et al., 2004), and modecoupling theory (Novikov and Sokolov, 2003).

\section{B. The thermodynamic connection}

The entropy model relates dynamics to thermodynamics: The larger the temperature variation of the configurational entropy is, the more fragile the liquid is. Since the specific heat is given by $c=d S / d \ln T$, the entropy model predicts that fragile liquids have a larger configurational contribution to the specific heat than strong liquids. If the identification $S_{\text {conf }} \cong S_{\text {exc }}$ is accepted, fragile liquids should have larger excess specific heat than strong liquids. This is traditionally reported as the general trend (Angell, 1985; Mohanty, 1995; Ito, Moynihan, and Angell, 1999; Xia and Wolynes, 2000), but even this conventional wisdom has recently been challenged (Ngai and Yamamuro, 1999; Huang and McKenna, 2001).

As mentioned, the identification $S_{\text {conf }} \cong S_{\text {exc }}$ is problematic. This brings into question the experimental support for the entropy model, but why then do fragility and excess heat capacity appear to correlate? The good news is that elastic models give rise to a correlation of this kind (Granato, 2002). To see this, consider first a one-dimensional harmonic oscillator $U(x)=U_{0}$ $+(1 / 2) m \omega_{0}^{2} x^{2}$. The entropy is the logarithm of the statistical mechanical "uncertainty" $\sqrt{\left\langle x^{2}\right\rangle}$ : in one dimension $S=k_{B} \ln \left(\sqrt{\left\langle x^{2}\right\rangle}\right)+$ const. Since $c=d S / d \ln T$ we find for the vibrational specific heat $2 c_{\mathrm{vib}} / k_{B}=d \ln \left\langle x^{2}\right\rangle / d \ln T$. For the crystalline state one expects almost temperature-independent elastic constants, implying that $2 c_{\text {cryst }} / k_{B}=1$, so the excess vibrational specific heat is given by $2 c_{\text {exc,vib }} / k_{B}=d \ln \left(\left\langle x^{2}\right\rangle / T\right) / d \ln T$. From Eqs. (8) and (11) we conclude that, since the temperature dependence of $a$ is insignificant,

$$
\frac{c_{\text {exc,vib }}}{c_{\text {cryst }}}=I .
$$

Thus elastic models predict that fragile liquids have larger excess vibrational specific heat than strong liquids.

The bad news is that the numbers do not fit experiment. The measured excess heat capacity also has a configurational contribution, so the calculation implies that $c_{\text {exc }} / c_{\text {cryst }}>I$. As an example, for ortho-terphenyl an excess heat capacity is predicted that is at least twice as large as $c_{\text {cryst }}$, whereas the measured number is below 1 . 
Generally, Eq. (16) predicts a too large excess heat capacity. In conclusion, the single-force-constant model is too simple to account for experiment.

This means that the elastic models are not strictly equivalent. The overwhelming contribution to the vibrational specific heat, however, comes from excitations with wavelength comparable to $a$, whereas, e.g., $c_{\infty}$ and $G_{\infty}$ are dominated by long-wavelength excitations. Thus it is possible that the phonon dispersion relation is slightly "deformed" at high frequencies upon cooling, which might explain the discrepancy. Such effects of temperature changes are well known, even for single crystals (Wallace, 1972).

\section{Relation to point-defect thermodynamics and solid-state diffusion}

Because the single-force-constant model is too simple, one cannot realistically expect that $G_{\infty} \propto K_{\infty}$ in their temperature variations. This brings into question how accurate the proportionalities of Eq. (15) are.

Interestingly, by far the most important temperature variation is that of the shear modulus: To calculate $\left\langle x^{2}\right\rangle$ one expands the vibrations on a complete set of normal modes. In a disordered structure these are not phonons with a well-defined wavelength and direction of propagation (Binder and Kob, 2005). Nevertheless, as a first approximation one might imagine describing the meansquare displacement in terms of transverse and longitudinal waves. Because there are twice as many transverse as longitudinal waves, the harmonic approximation Eq. (11) implies that $1 / \Delta E \propto 2 / G_{\infty}+1 /\left(K_{\infty}+4 G_{\infty} / 3\right)$. From this it can be shown that, if temperature variations are quantified in terms of log-log derivatives, at least $92 \%$ of the index $I$ derives from the temperature variation of $G_{\infty}$ and at most $8 \%$ from that of $K_{\infty}$ (Dyre and Olsen, 2004). Thus whether or not $G_{\infty}(T)$ and $K_{\infty}(T)$ are proportional, Eq. (15) applies to a good approximation if $c_{\infty}$ is taken to be the high-frequency transverse sound velocity. The dominance of the shear modulus ${ }^{9}$ in the harmonic approximation comes from the following three factors: (1) There are two transverse phonons for each longitudinal one. (2) $G_{\infty}$ plays a role also for the longitudinal phonons. (3) Longitudinal phonons are associated with larger elastic constants than the transverse, thus giving less than $1 / 3$ contribution to $\left\langle x^{2}\right\rangle$.

Elastic models involve assumptions and reasoning which have a long history in the field of point defects in crystals (Flynn, 1968; Teodosiu, 1982; Varotsos and Alexopoulos, 1986; Alexander, 1998; Kraftmakher, 1998; Faupel et al., 2003); the only new thing is the unusually strong temperature variation of the high-frequency elastic constants of viscous liquids. For instance, the Zener

\footnotetext{
9"Shear dominance" appears to be a general rule according to which, whenever a physical quantity depends on both bulk and shear moduli, the dependence of the shear modulus is the most important (Rehn et al., 1974; Johnson, 1988; Granato, 1992; Dyre, 2004a).
}

(1942) strain model assumes that most of the solid defect energy is elastic and located in its surroundings, and the shoving model derivation is similar to that of the Anderson-Stuart (1954) theory of ionic conduction in solids. A futher connection to conventional solid-state defect theory comes from noting that vacancy-formation enthalpies scale with the melting temperature (Doyama and Koehler, 1976), and that these enthalpies are proportional to the bulk modulus (Varotsos and Alexopoulos, 1986). When combined with the elastic model prediction that $T_{g}$ scales with the glass moduli and the empirical fact that glass and crystal moduli are usually similar, elastic models imply that the glass transition temperature must scale with the melting temperature. This applies generally, as discussed already in connection with the glass transition Lindemann criterion.

\section{RECENT DEVELOPMENTS}

The last few years have revealed unexpected connections between the fast and slow degrees of freedom of glass-forming liquids. Whether or not these new findings are eventually explained in terms of elastic models, they do serve to emphasize that the starting point of these models-the fast-slow connection-is not unreasonable.

Scopigno et al. (2003) reported a correlation between (liquid) fragility and properties of the glassy phase. These authors used inelastic x-ray scattering, a technique whereby energy changes of $\mathrm{x}$ rays $(\mathrm{keV})$ are measured with $\mathrm{meV}$ resolution. If the Brillouin line intensity is integrated over frequency, the ratio of the central line contribution to the entire intensity at long wavelengths and low temperatures may be written as $1 /\left(1+\alpha T / T_{g}\right)$. Scopigno et al. found that $\alpha$ is proportional to the fragility. Buchenau and Wischnewski (2004) noted that if the above expression applies up to $T_{g}, \alpha$ equals the ratio between the vibrational (short-time) and relaxational (long-time) contributions to the compressibility of the liquid phase just above $T_{g}$. Since the compressibility is a measure of density fluctuations (Hansen and McDonald, 1986; Barrat and Hansen, 2003), the larger the fragility is, the larger is the fraction of vibrational to relaxational density fluctuations in the equilibrium liquid.

Novikov and Sokolov (2004) showed that the glass Poisson ratio correlates with the liquid fragility (Dyre, 2004b). Recall that Poisson's ratio is the ratio of transverse expansion to longitudinal contraction when a solid is compressed in one direction. Most materials have a Poisson ratio of between 0, for which no lateral expansion occurs, and 0.5 , for which the volume is kept constant. Cork has a Poisson ratio close to 0 (which conveniently implies that there is little lateral expansion when a wine bottle is corked), most steels are around 0.3, and rubber is close to 0.5 . The Poisson ratio is larger the larger $K / G$ is. It now appears that for glasses $K / G$ is larger, the larger the fragility of the glass-forming liquid is. At the glass transition the structure freezes and the high-frequency elastic constants $K_{\infty}$ and $G_{\infty}$ transform into the glass elastic constants $K$ and $G$. Thus the 
Novikov-Sokolov correlation may be formulated as an observation referring only to the liquid phase, namely, that fragility correlates with $K_{\infty} / G_{\infty}$ (Novikov, Ding, and Sokolov, 2005).

Elastic models deal with equilibrium viscous liquid dynamics, but the idea that the activation energy is proportional to an elastic modulus may also be applied to describe aging below $T_{g}$ (Olsen, Dyre, and Christensen, 1998). Granato and Khonik (2004) formulated a theory for aging of bulk metallic glasses based on $\Delta E \propto G$, where $G$ is the glass shear modulus. This theory explains the observation that during aging the effective glass viscosity increases as a linear function of time $\eta_{\text {eff }}=A+B t$. Johnson and Samwer (2005) developed a model for plastic yielding of metallic glasses where the activation energy for a "shear transformation zone" depends on the shear stress $\sigma$ and goes to zero at a critical stress $\sigma_{c}$ in the following way: $\Delta E \propto G\left(\sigma_{c}-\sigma\right)^{3 / 2}$. This expression generalizes Eq. (12) to deal with plastic flow. Experiments on 30 bulk metallic glasses confirm the model.

\section{OUTLOOK}

Contrary to what is often stated, the liquid-glass transition is really no big mystery - it is a falling out of equilibrium of a kind that inevitably happens to any system cooled faster than its temperature-dependent internal equilibration time. The scientific challenge is to understand the viscous liquid phase above $T_{g}$.

It is not obvious that any universal theory for the temperature dependence of the viscous liquid relaxation time exists. And if such a theory does exist, it is not obvious that the activation energy relates to any macroscopic quantity. Nevertheless, these two features are shared by four classes of phenomenological models, where the stronger than Arrhenius behavior is a consequence of the fact that

(1) for the entropy model, the configurational entropy decreases with decreasing temperature;

(2) for free-volume models, the free volume decreases with decreasing temperature;

(3) for energy-based models, the energy decreases with decreasing temperature;

(4) for elastic models, the short-time elastic constants increase with decreasing temperature.

For the first three model classes the activation energy is controlled by a difference of quantities; this is the only possibility for deriving activation energies that vary sufficiently with temperature from $S(T), V(T)$, or $E(T)$. For elastic models there is no subtraction in the expression for the activation energy.

Elastic models are conceptually related to the freevolume models through the assumption that the activation energy is determined by a physical quantity which can be measured on a short time scale (configurational entropy obviously cannot be determined by a fast measurement). Moreover, several elastic models are based on the free-volume assumption that a temporary local density decrease is needed for a flow event. On the other hand, elastic models have the property that fragility correlates with the excess specific heat in common with the entropy model.

In elastic models the fragility and excess vibrational specific heat are larger, the more nonlinear the vibrational $\left\langle x^{2}\right\rangle$ is as a function of temperature, i.e., the more anharmonic the system is on the short time scale. Thus vibrational anharmonicity (Angell, 1995) is a central ingredient of elastic models. At high pressure one expects the potential to become less anharmonic, so elastic models are consistent with the finding that fragility generally decreases with increasing pressure (Casalini and Roland, 2005; Roland et al., 2005).

It is too early to tell whether or not elastic models give the basically correct explanation of the non-Arrhenius viscosity. If this is the case, there are several notable conclusions:

- Viscous liquid dynamics relate to the broad, classical branches "solid-state diffusion" and "thermodynamics of point defects in solids."

- Viscous liquid slowing down is not a manifestation of the approach to a nearly avoided or unreachable phase transition where the relaxation time becomes infinite, and the slowing down is not caused by a diverging length scale.

- Similarly, the liquid-glass transition has little in common with either ergodic-nonergodic transitions of various lattice models (Pitts, Young, and Andersen, 2000) and ideal mode-coupling theory, or with the jamming transition of granular media (D'Anna and Gremaud, 2001).

- The activation energy controlling aging of the glassy phase may be monitored directly, e.g., by highfrequency sound velocity measurements. This could make it easier to predict and control glass annealing.

For a number of reasons the study of viscous liquids approaching the glass transition is likely to remain an exciting branch of condensed-matter physics for years to come: (1) the most basic problems are unsolved; (2) computers are still too slow for the secrets of viscous liquid dynamics to be revealed by brute force simulations; and (3) there is a small, dimensionless number characterizing viscous liquids just above the glass transition $\left(\sim 10^{-30}\right)$, indicating that a simple and general theory for viscous liquid dynamics may exist. Although the research field is still open to new theoretical developments and even simple phenomenological modeling, future breakthroughs are likely to come from careful and accurate experiments.

\section{ACKNOWLEDGMENTS}

The author wishes to thank Austen Angell, Nick Bailey, Uli Buchenau, Shankar Das, Gregor Diezemann, Andy Granato, Gyan Johari, Kristine Niss, and Thomas Schrøder for many suggestions improving the manu- 
script. This work was supported by the Danish National Research Foundation's Centre for Viscous Liquid Dynamics "Glass and Time."

\section{REFERENCES}

Adam, G., and J. H. Gibbs, 1965, "On the temperature dependence of cooperative relaxation properties in glass-forming liquids," J. Chem. Phys. 43, 139-146.

Alba-Simionesco, C., 2001, "Salient properties of glassforming liquids close to the glass transition," C. R. Acad. Sci., Ser IV: Phys., Astrophys. 2, 203-216.

Alba-Simionesco, C., A. Cailliaux, A. Alegria, and G. Tarjus, 2004, "Scaling out the density dependence of the $\alpha$ relaxation in glass-forming polymers," Europhys. Lett. 68, 58-64.

Alexander, S., 1998, "Amorphous solids: Their structure, lattice dynamics and elasticity," Phys. Rep. 296, 65-236.

Anderson, O. L., and D. A. Stuart, 1954, "Calculation of activation energy of ionic conductivity in silica glasses by classical methods," J. Am. Ceram. Soc. 37, 573-580.

Anderson, P. W., 1979, "Lectures on amorphous systems," in Ill-Condensed Matter, edited by R. Balian, R. Maynard, and G. Toulouse (North-Holland, Amsterdam), pp. 159-261.

Angell, C. A., 1968, “Oxide glasses in light of the 'ideal glass' concept: I. Ideal and nonideal transitions, and departures from ideality," J. Am. Ceram. Soc. 51, 117-124.

Angell, C. A., 1985, "Strong and fragile liquids," in Relaxations in Complex Systems, edited by K. L. Ngai and G. B. Wright (U.S. GPO, Washington, D.C.), pp. 3-11.

Angell, C. A., 1991, "Relaxation in liquids, polymers and plastic crystals - strong/fragile patterns and problems," J. NonCryst. Solids 131, 13-31.

Angell, C. A., 1995, "Formation of glasses from liquids and biopolymers," Science 267, 1924-1935.

Angell, C. A., 1997, "Entropy and fragility in supercooling liquids,” J. Res. Natl. Inst. Stand. Technol. 102, 171-185.

Angell, C. A., K. L. Ngai, G. B. McKenna, P. F. McMillan, and S. W. Martin, 2000, "Relaxation in glassforming liquids and amorphous solids," J. Appl. Phys. 88, 3113-3157.

Angell, C. A., and K. J. Rao, 1972, "Configurational excitations in condensed matter, and the 'bond lattice' model for the liquid-glass transition,” J. Chem. Phys. 57, 470-481.

Arrhenius, S., 1889, "Über die Reaktionsgeschwindigkeit bei der Inversion von Rohrzucker durch Säuren," Z. Phys. Chem. 4, 226-248.

Avramov, I., 1996, "Kinetics of structural relaxation of glassforming melts," Thermochim. Acta 280, 363-382.

Avramov, I., 2005, "Viscosity in disordered media," J. NonCryst. Solids 351, 3163-3173.

Barlow, A. J., J. Lamb, A. J. Matheson, P. R. K. L. Padmini, and J. Richter, 1967, "Viscoelastic relaxation of supercooled liquids. I.," Proc. R. Soc. London, Ser. A 298, 467-480.

Barrat, J.-L., and J.-P. Hansen, 2003, Basic Concepts for Simple and Complex Liquids (Cambridge University Press, Cambridge, England).

Barton, A. F. M., 1972, "The structural component of acoustic absorption in liquids," Adv. Mol. Relax. Processes 4, 87-137.

Bässler, H., 1987, "Viscous flow in supercooled liquids analyzed in terms of transport theory for random media with energetic disorder," Phys. Rev. Lett. 58, 767-770.

Bellissent, R., and G. Tourand, 1980, "Short range order in amorphous and liquid $\mathrm{Se}_{1-x} \mathrm{Te}_{x}$ systems," J. Non-Cryst. Solids 35, 1221-1226.
Berry, R. S., and B. M. Smirnov, 2005, "Phase transitions and adjacent phenomena in simple atomic systems," Phys. Usp. 48, 345-388.

Binder, K., and W. Kob, 2005, Glassy Materials and Disordered Solids: An Introduction to their Statistical Mechanics (World Scientific, Singapore).

Birge, N. O., and S. R. Nagel, 1985, "Specific-heat spectroscopy of the glass transition," Phys. Rev. Lett. 54, 2674-2677.

Bisquert, J., 2005, "Master equation approach to the nonequilibrium negative specific heat at the glass transition," Am. J. Phys. 73, 735-741.

Böhmer, R., G. Diezemann, G. Hinze, and E. Rössler, 2001, "Dynamics of supercooled liquids and glassy solids," Prog. Nucl. Magn. Reson. Spectrosc. 39, 191-267.

Böhmer, R., K. L. Ngai, C. A. Angell, and D. J. Plazek, 1993, "Nonexponential relaxations in strong and fragile glass formers," J. Chem. Phys. 99, 4201-4209.

Boon, J. P., and S. Yip, 1980, Molecular Hydrodynamics (McGraw-Hill, New York).

Bordat, P., F. Affouard, M. Descamps, and K. L. Ngai, 2004, "Does the interaction potential determine both the fragility of a liquid and the vibrational properties of its glassy state?," Phys. Rev. Lett. 93, 105502.

Bouchaud, J. P., 1992, "Weak ergodicity breaking and aging in disordered systems," J. Phys. I 2, 1705-1713.

Brawer, S., 1985, Relaxation in Viscous Liquids and Glasses (American Ceramic Society, Columbus, OH).

Brawer, S. A., 1984, "Theory of relaxation in viscous liquids and glasses," J. Chem. Phys. 81, 954-975.

Brush, S. G., 1962, "Theories of liquid viscosity," Chem. Rev. (Washington, D.C.) 62, 513-548.

Buchenau, U., and A. Wischnewski, 2004, "Fragility and compressibility at the glass transition," Phys. Rev. B 70, 092201.

Buchenau, U., and R. Zorn, 1992, "A relation between fast and slow motions in glassy and liquid selenium," Europhys. Lett. 18, 523-528.

Bueche, F., 1959, "Mobility of molecules in liquids near the glass temperature," J. Chem. Phys. 30, 748-752.

Casalini, R., and C. M. Roland, 2004, "Thermodynamical scaling of the glass transition dynamics," Phys. Rev. E 69, 062501. Casalini, R., and C. M. Roland, 2005, "Scaling of the supercooled dynamics and its relation to the pressure dependences of the dynamic crossover and the fragility of glass formers," Phys. Rev. B 71, 014210.

Cavagna, A., A. Attanasi, and J. Lorenzana, 2005, "Viscoelasticity and metastability limit in supercooled liquids," Phys. Rev. Lett. 95, 115702.

Chandler, D., J. D. Weeks, and H. C. Andersen, 1983, "Van der Waals picture of liquids, solids, and phase transformations," Science 220, 787-794.

Christensen, T., 1985, "The frequency dependence of the specific heat at the glass transition," J. Phys. (Paris), Colloq. 46 (C8), 635-637.

Christensen, T., and N. B. Olsen, 1994, "Determination of the frequency-dependent bulk modulus of glycerol using a piezoelectric spherical shell,” Phys. Rev. B 49, 15396-15399.

Christensen, T., and N. B. Olsen, 1995, "A rheometer for the measurement of a high shear modulus covering more than seven decades of frequency below $50 \mathrm{kHz}$," Rev. Sci. Instrum. 66, 5019-5031.

Cohen, M. H., and D. Turnbull, 1959, "Molecular transport in liquids and glasses,” J. Chem. Phys. 31, 1164-1169.

Corezzi, S., L. Comez, and D. Fioretto, 2004, "Can experi- 
ments select the configurational component of excess entropy?," Eur. Phys. J. E 14, 143-147.

Cornicchi, E., G. Onori, and A. Paciaroni, 2005, "Picosecondtime-scale fluctuations of proteins in glassy matrices: The role of viscosity,” Phys. Rev. Lett. 95, 158104.

Cotterill, R., 1985, The Cambridge Guide to the Material World (Cambridge University Press, Cambridge, England).

D'Anna, G., and G. Gremaud, 2001, "The jamming route to the glass state in weakly perturbed granular media," Nature (London) 413, 407-409.

Das, S. P., 2004, "Mode-coupling theory and the glass transition in supercooled liquids," Rev. Mod. Phys. 76, 785-851.

Debenedetti, P. G., 1996, Metastable Liquids: Concepts and Principles (Princeton University Press, Princeton, NJ).

Debenedetti, P. G., and F. H. Stillinger, 2001, "Supercooled liquids and the glass transition," Nature (London) 410, 259267.

Diezemann, G., 1997, “A free-energy landscape model for primary relaxation in glass-forming liquids: Rotations and dynamic heterogeneities," J. Chem. Phys. 107, 10112-10120.

Diezemann, G., 2005, “Aging in a free-energy landscape model for glassy relaxation," J. Chem. Phys. 123, 204510.

Diezemann, G., H. Sillescu, G. Hinze, and R. Böhmer, 1998, "Rotational correlation functions and apparently enhanced translational diffusion in a free-energy landscape model for the alpha relaxation in glass-forming liquids," Phys. Rev. E 57, 4398-4410.

Donth, E., 2001, The Glass Transition (Springer, Berlin).

Doolittle, A. K., 1951, "Studies in Newtonian flow. II. The dependence of the viscosity of liquids on free-space," J. Appl. Phys. 22, 1471-1475.

Doremus, R. H., 1994, Glass Science, 2nd ed. (Wiley, New York).

Douglas, J. F., and D. Leporini, 1998, "Obstruction model of the fractional Stokes-Einstein relation in glass-forming liquids," J. Non-Cryst. Solids 235, 137-141.

Doyama, M., and J. S. Koehler, 1976, "The relation between formation energy of a vacancy and the nearest neighbor interactions in pure metals and liquid metals," Acta Metall. 24, 871-879.

Dreyfus, C., and R. M. Pick, 2001, "Relaxations and vibrations in supercooled liquids," C. R. Acad. Sci., Ser IV: Phys., Astrophys. 2, 217-237.

Dushman, S., 1920, "Theory of unimolecular reaction velocities," J. Franklin Inst. 189, 515-518.

Dyre, J. C., 1987, "Master-equation approach to the glass transition," Phys. Rev. Lett. 58, 792-795.

Dyre, J. C., 1995, "Energy master equation: A lowtemperature approximation to Bässler's random-walk model," Phys. Rev. B 51, 12276-12294.

Dyre, J. C., 1998, "Source of non-Arrhenius average relaxation time in glass-forming liquids," J. Non-Cryst. Solids 235, 142149.

Dyre, J. C., 1999, "Solidity of viscous liquids," Phys. Rev. E 59, 2458-2459.

Dyre, J. C., 2004a, "Shear dominance of point defect energies in solids," e-print cond-mat/0406759.

Dyre, J. C., 2004b, "Glasses: Heirs of liquid treasures," Nat. Mater. 3, 749-750.

Dyre, J. C., 2005, "Solidity of viscous liquids. III. $\alpha$ relaxation," Phys. Rev. E 72, 011501.

Dyre, J. C., 2006, "Solidity of viscous liquids. IV. Density fluctuations," Phys. Rev. E 74, 021502.
Dyre, J. C., and N. B. Olsen, 2004, "Landscape equivalent of the shoving model," Phys. Rev. E 69, 042501.

Dyre, J. C., N. B. Olsen, and T. Christensen, 1996, "Local elastic expansion model for viscous-flow activation energies of glass-forming molecular liquids," Phys. Rev. B 53, 2171-2174. Dzhalilov, S. U., and K. I. Rzaev, 1967, "On the phenomenon of selenium vitrification," Phys. Status Solidi 20, 261-266.

Edwards, S. F., 1976, “Theory of glasses," Polymer 17, 933-937. Elliott, S. R., 1990, Physics of Amorphous Materials, 2nd ed. (Longman Scientific, London).

Eyring, H., 1936, "Viscosity, plasticity, and diffusion as examples of absolute reaction rates," J. Chem. Phys. 4, 283-291.

Faupel, F., W. Frank, M. P. Macht, H. Mehrer, V. Naundorf, K. Ratzke, H. R. Schober, S. K. Sharma, and H. Teichler, 2003, "Diffusion in metallic glasses and supercooled melts," Rev. Mod. Phys. 75, 237-280.

Flynn, C. P., 1968, "Atomic migration in monatomic crystals," Phys. Rev. 171, 682-698.

Franks, F., 2003, "Scientific and technological aspects of aqeous glasses," Biophys. Chem. 105, 251-261.

Fredrickson, G. H., 1988, "Recent developments in dynamical theories of the liquid-glass transition," Annu. Rev. Phys. Chem. 39, 149-180.

Fulcher, G. S., 1925, "Analysis of recent measurements of the viscosity of glasses," J. Am. Ceram. Soc. 8, 339-355.

Garrahan, J. P., and D. Chandler, 2002, "Geometrical explanation and scaling of dynamical heterogeneities in glass forming systems," Phys. Rev. Lett. 89, 035704.

Gibbs, J. H., and E. A. DiMarzio, 1958, "Nature of the glass transition and the glassy state," J. Chem. Phys. 28, 373-383.

Goldenfeld, N., 1992, Lectures on Phase Transitions and the Renormalization Group (Addison-Wesley, Reading, MA).

Goldstein, M., 1969, "Viscous liquids and the glass transition: A potential energy barrier picture," J. Chem. Phys. 51, 37283739.

Goldstein, M., 1972, "Glass and other relaxations in liquids," Faraday Symp. Chem. Soc. 6, 7-13.

Goldstein, M., 1976, "Viscous liquids and the glass transition. V. Sources of the excess specific heat of the liquid," J. Chem. Phys. 64, 4767-4774.

Götze, W., and L. Sjögren, 1992, "Relaxation processes in supercooled liquids,” Rep. Prog. Phys. 55, 241-376.

Granato, A. V., 1992, "Interstitialcy model for condensed matter states of face-centered-cubic metals," Phys. Rev. Lett. 68, 974-977.

Granato, A. V., 1999, "A relation between fragility, specific heat, and shear modulus temperature dependence for simple supercooled liquids," in Bulk Metallic Glasses, edited by W. L. Johnson, C. T. Liu, and A. Inoue, MRS Symposia Proceedings No. 554 (Materials Research Society, Pittsburgh), pp. 299-303.

Granato, A. V., 2002, "The specific heat of simple liquids," J. Non-Cryst. Solids 307, 376-386.

Granato, A. V., and V. A. Khonik, 2004, "An interstitialcy theory of structural relaxation and related viscous flow of glasses," Phys. Rev. Lett. 93, 155502.

Greet, R. J., and D. Turnbull, 1967, "Test of Adam-Gibbs liquid viscosity model with o-terphenyl specific-heat data," J. Chem. Phys. 47, 2185-2190.

Grest, G. S., and M. H. Cohen, 1981, "Liquids, glasses, and the glass transition: A free-volume approach," in Advances in Chemical Physics, edited by I. Prigogine and S. A. Rice (Wiley, New York), Vol. 48, pp. 455-525. 
Hall, R. W., and P. G. Wolynes, 1987, "The aperiodic crystal picture and free energy barriers in glasses," J. Chem. Phys. 86, 2943-2948.

Hansen, J. P., and I. R. McDonald, 1986, Theory of Simple Liquids, 2nd ed. (Academic, New York).

Harrison, G., 1976, The Dynamic Properties of Supercooled Liquids (Academic, New York).

Heuer, A., and H. W. Spiess, 1994, "Universality of the glass transition temperature," J. Non-Cryst. Solids 176, 294-298.

Hodge, I. M., 1994, "Enthalpy relaxation and recovery in amorphous materials," J. Non-Cryst. Solids 169, 211-266.

Huang, D., and G. B. McKenna, 2001, "New insights into the fragility dilemma in liquids," J. Chem. Phys. 114, 5621-5630.

Ito, K., C. T. Moynihan, and C. A. Angell, 1999, "Thermodynamic determination of fragility in liquids and a fragile-tostrong liquid transition in water," Nature (London) 398, 492 495.

Jakobsen, B., K. Niss, and N. B. Olsen, 2005, "Dielectric and shear mechanical alpha and beta relaxation in seven glassforming liquids," J. Chem. Phys. 123, 234511.

Johari, G. P., 1974, "Introduction to the glassy state in the undergraduate curriculum," J. Chem. Educ. 51, 23-27.

Johari, G. P., 2002, "The entropy loss on supercooling a liquid and anharmonic contributions," J. Chem. Phys. 116, 20432046.

Johari, G. P., 2003, "Simple ratio for testing a supercooled liquid's relaxation time-entropy relation,” J. Phys. Chem. B 107, 5048-5051.

Johari, G. P., and M. Goldstein, 1970, "Viscous liquids and the glass transition. II. Secondary relaxations in glasses of rigid molecules," J. Chem. Phys. 53, 2372-2388.

Johari, G. P., and D. Pyke, 2000, "On the glassy and supercooled liquid states of a common medicament: Aspirin," Phys. Chem. Chem. Phys. 2, 5479-5484.

Johnson, R. A., 1988, "Relationship between defect energies and embedded-atom-method parameters," Phys. Rev. B 37, 6121-6125.

Johnson, W. L., and K. Samwer, 2005, “A universal criterion for plastic yielding of metallic glasses with a $\left(T / T_{g}\right)^{2 / 3}$ temperature dependence," Phys. Rev. Lett. 95, 195501.

Kanaya, T., T. Tsukushi, K. Kaji, J. Bartos, and J. Kristiak, 1999, "Microscopic basis of free-volume concept as studied by quasielastic neutron scattering and positron annihilation lifetime spectroscopy,” Phys. Rev. E 60, 1906-1912.

Kasapis, S., 2005, "Glass transition phenomena in dehydrated model systems and foods: A review," Drying Technol. 23, 731-757.

Kauzmann, W., 1948, "The nature of the glassy state and the behavior of liquids at low temperatures," Chem. Rev. (Washington, D.C.) 43, 219-256.

Kirkpatrick, T. R., D. Thirumalai, and P. G. Wolynes, 1989, "Scaling concepts for the dynamics of viscous liquids near an ideal glassy state," Phys. Rev. A 40, 1045-1054.

Kittel, C., 1996, Introduction to Solid State Physics, 7th ed. (Wiley, New York).

Kivelson, D., S. A. Kivelson, X. Zhao, Z. Nussinov, and G. Tarjus, 1995, "A thermodynamic theory of supercooled liquids," Physica A 219, 27-38.

Kivelson, D., G. Tarjus, X. Zhao, and S. A. Kivelson, 1996, "Fitting of viscosity: Distinguishing the temperature dependences predicted by various models of supercooled liquids," Phys. Rev. E 53, 751-758.

Kob, W., 2004, "Supercooled liquids, the glass transition, and computer simulations," in Slow Relaxations and Nonequilibrium Dynamics in Condensed Matter, Proceedings of the Les Houches Summer School of Theoretical Physics, Session LXXVII, 2002, edited by J.-L. Barrat, M. Feigelman, J. Kurchan, and J. Dalibard (Springer, Berlin), pp. 199-269.

Köhler, U., and C. Herzig, 1988, "On the correlation between self-diffusion and the low-frequency LA 2/3(111) phonon mode in BCC metals," Philos. Mag. A 58, 769-786.

Kovacs, A. J., 1963, "Transition vitreuse dans les polymères amorphes. Etude phénoménologique," Fortschr. Hochpolym.-Forsch. 3, 394-507.

Kraftmakher, Y., 1998, "Equilibrium vacancies and thermophysical properties of metals," Phys. Rep. 299, 79-188.

Kramers, H. A., 1940, "Brownian motion in a field of force and the diffusion model of chemical reactions," Physica (Amsterdam) 7, 284-304.

Kremer, F., and A. Schönhals, 2002, Eds., Broadband Dielectric Spectroscopy (Springer, Berlin).

Kudlik, A., S. Benkhof, T. Blochowicz, C. Tschirwitz, and E. Rössler, 1999, "The dielectric response of simple organic glass formers," J. Mol. Struct. 479, 201-218.

Lamb, J., 1978, "Viscoelasticity and lubrication: A review of liquid properties," J. Rheol. 22, 317-347.

Landau, L. D., and E. M. Lifshitz, 1970, Theory of Elasticity, 2nd ed. (Pergamon, London).

Le Meste M., D. Champion, G. Roudaut, G. Blond, and D. Simatos, 2002, "Glass transition and food technology: A critical appraisal,” J. Food. Sci. 67, 2444-2458.

Levine, H., 2002, Ed., Amorphous Food and Pharmaceutical Systems (Royal Society of Chemistry, London).

Lindemann, F. A., 1910, "Über die Berechnung molekularer Eigenfrequenzen," Phys. Z. 11, 609-612.

Litovitz, T. A., 1959, "Ultrasonic spectroscopy in liquids," J. Acoust. Soc. Am. 31, 681-691.

Löffler, J. F., 2003, "Bulk metallic glasses,” Intermetallics 11, 529-540.

Lunkenheimer, P., U. Schneider, R. Brand, and A. Loidl, 2000, "Glassy dynamics," Contemp. Phys. 41, 15-36.

Magazu, S., G. Maisano, F. Migliardo, and C. Mondelli, 2004, "Mean-square displacement relationship in bioprotectant systems by elastic neutron scattering," Biophys. J. 86, 3241-3249. Malinovsky, V. K., and V. N. Novikov, 1992, "The nature of the glass transition and the excess low-energy density of vibrational states in glasses," J. Phys.: Condens. Matter 4, L139_ L143.

Martinez, L.-M., and C. A. Angell, 2001, "A thermodynamic connection to the fragility of glass-forming liquids," Nature (London) 410, 663-667.

Matyushov, D. V., and C. A. Angell, 2005, "Two-Gaussian excitations model for the glass transition," J. Chem. Phys. 123, 034506.

Maxwell, J. C., 1867, "On the dynamical theory of gases," Philos. Trans. R. Soc. London 157, 49-88.

Mazurin, O. V., 1977, "Relaxation phenomena in glass," J. Non-Cryst. Solids 25, 129-169.

Mohanty, U., 1995, "Supercooled liquids," in Advances in Chemical Physics, edited by I. Prigogine and S. A. Rice (Wiley, New York), Vol. 89, pp. 89-158.

Mooney, M., 1957, "A theory of the viscosity of a Maxwellian elastic liquid,” Trans. Soc. Rheol. 1, 63-94.

Mortensen, K., W. Brown, and B. Nordén, 1992, "Inverse melting transition and evidence of three-dimensional cubatic structure in a block-copolymer micellar system," Phys. Rev. 
Lett. 68, 2340-2343

Moynihan, C. T., A. J. Easteal, J. Wilder, and J. Tucker, 1974, "Dependence of the glass transition temperature on heating and cooling rate," J. Phys. Chem. 78, 2673-2677.

Nemilov, S. V., 1968, "Kinetics of elementary processes in the condensed state. II. Shear relaxation and the equation of state for solids," Russ. J. Phys. Chem. 42, 726-729.

Nemilov, S V., 1978, "Valence configurational theory and its experimental basis for viscous flow in supercooled glassforming liquids," Sov. J. Glass Phys. Chem. 4, 113-129.

Nemilov, S. V., 1992, "Viscous flow of glasses correlated with their structure. Application of the rate theory," Sov. J. Glass Phys. Chem. 18, 1-27.

Nemilov, S. V., 1995, Thermodynamic and Kinetic Aspects of the Vitreous State (CRC, Boca Raton, FL).

Ngai, K. L., 2004, "Why the fast relaxation in the picosecond to nanosecond time range can sense the glass transition," Philos. Mag. 84, 1341-1353.

Ngai, K. L., and O. Yamamuro, 1999, "Thermodynamic fragility and kinetic fragility in supercooling liquids: A missing link in molecular liquids," J. Chem. Phys. 111, 10403-10406.

Novikov, V. N., Y. Ding, and A. P. Sokolov, 2005, "Correlation of fragility of supercooled liquids with elastic properties of glasses," Phys. Rev. E 71, 061501.

Novikov, V. N., E. Rössler, V. K. Malinovsky, and N. V. Surovtsev, 1996, "Strong and fragile liquids in percolation approach to the glass transition," Europhys. Lett. 35, 289-294.

Novikov, V. N., and A. P. Sokolov, 2003, "Universality of the dynamic crossover in glass-forming liquids: A 'magic' relaxation time," Phys. Rev. E 67, 031507.

Novikov, V. N., and A. P. Sokolov, 2004, "Poisson's ratio and the fragility of glass-forming liquids," Nature (London) 431, 961-963.

Olsen, N. B., J. C. Dyre, and T. Christensen, 1998, "Structural relaxation monitored by instantaneous shear modulus," Phys. Rev. Lett. 81, 1031-1033.

Onuchic, J. N., Z. Luthey-Schulten, and P. G. Wolynes, 1997, "Theory of protein folding: The energy landscape perspective," Annu. Rev. Phys. Chem. 48, 545-600.

Owen, A. E., 1985, "The glass transition," in Amorphous Solids and the Liquid State, edited by N. H. March, R. A. Street, and M. Tosi (Plenum, New York), pp. 395-432.

Pedersen, U. R., T. Hecksher, J. C. Dyre, and T. B. Schrøder, 2006, "An energy landscape model for glass-forming liquids in three dimensions," e-print cond-mat/0511147, J. NonCryst. Solids (to be published).

Petrie, F., 1925, "Glass found in Egypt," Trans. British Newcomen Soc. 5, 72-76.

Phillips, J. C., 1996, "Stretched exponential relaxation in molecular and electronic glasses," Rep. Prog. Phys. 59, 1133 1207.

Phillips, W. A., U. Buchenau, N. Nücker, A.-J. Dianoux, and W. Petry, 1989, "Dynamics of glassy and liquid selenium," Phys. Rev. Lett. 63, 2381-2384.

Pimpinelli, A., and J. Villain, 1998, Physics of Crystal Growth (Cambridge University Press, Cambridge, England).

Pitts, S. J., T. Young, and H. C. Andersen, 2000, "Facilitated spin models, mode coupling theory, and ergodic-nonergodic transitions," J. Chem. Phys. 113, 8671-8679.

Plazek, D. J., and K. L. Ngai, 1991, “Correlation of polymer segmental chain dynamics with temperature-dependent timescale shifts," Macromolecules 24, 1222-1224.

Prevosto, D., M. Lucchesi, S. Capaccioli, R. Casalini, and P. A.
Rolla, 2003, "Correlation between configurational entropy and structural relaxation time in glass-forming liquids," Phys. Rev. B 67, 174202.

Rawson, H., 1988, "Glass and its history of service," IEE Proc., Part A: Phys. Sci., Meas. Instrum., Manage. Educ. 135, 325345.

Rehn, L. E., J. Holder, A. V. Granato, R. R. Coltman, and F. W. Young, 1974, "Effects of thermal-neutron irradiation on the elastic constants of copper," Phys. Rev. B 10, 349-362.

Ritort, F., and P. Sollich, 2003, "Glassy dynamics of kinetically constrained models," Adv. Phys. 52, 291-342.

Roland, C. M., S. Hensel-Bielowka, M. Paluch, and R. Casalini, 2005, "Supercooled dynamics of glass-forming liquids and polymers under hydrostatic pressure," Rep. Prog. Phys. 68, 1405-1478.

Roland, C. M., and K. L. Ngai, 1996, "The anomalous DebyeWaller factor and the fragility of glasses," J. Chem. Phys. 104, 2967-2970.

Ruocco, G., F. Sciortino, F. Zamponi, C. De Michele, and T. Scopigno, 2004, "Landscapes and fragilities," J. Chem. Phys. 120, 10666-10680.

Sanditov, D. S., and S. S. Sangadiev, 1998, "Condition of glass transition in the fluctuation free volume theory and the Lindemann criterion for melting," Glass Phys. Chem. 24, 285294.

Sanditov, D. S., S. S. Sangadiev, and G. V. Kozlov, 1998, “On the correlation between elastic modulus of vitreous solids and glass transition temperature of melts," Glass Phys. Chem. 24, 539-545.

Scherer, G. W., 1986, Relaxation in Glass and Composites (Wiley, New York).

Scherer, G. W., 1990, "Theories of relaxation," J. Non-Cryst. Solids 123, 75-89.

Schneider, U., P. Lunkenheimer, R. Brand, and A. Loidl, 1998, "Dielectric and far-infrared spectroscopy of glycerol," J. NonCryst. Solids 235, 173-179.

Schrøder, T. B., S. Sastry, J. C. Dyre, and S. C. Glotzer, 2000, "Crossover to potential energy landscape dominated dynamics in a model glass-forming liquid," J. Chem. Phys. 112, 9834-9840.

Schug, K. U., H. E. King, and R. Böhmer, 1998, "Fragility under pressure: Diamond anvil cell viscometry of orthoterphenyl and salol," J. Chem. Phys. 109, 1472-1477.

Schweizer, K. S., and E. J. Saltzman, 2004, “Activated hopping, barrier fluctuations, and heterogeneity in glassy suspensions and liquids," J. Phys. Chem. B 108, 19729-19741.

Sciortino, F., 2005, "Potential energy landscape description of supercooled liquids and glasses," J. Stat. Mech.: Theory Exp. P05015.

Scopigno, T., G. Ruocco, F. Sette, and G. Monaco, 2003, "Is the fragility of a liquid embedded in the properties of its glass?" Science 302, 849-852.

Sethna, J. P., 1988, "Speculations on the glass transition," Europhys. Lett. 6, 529-534.

Simon, F., 1930, "Fünfundzwanzig Jahre Nernstscher Wärmesatz," Ergeb. Exakten Naturwiss. 9, 222-274.

Simon, F., 1931, "Über den Zustand der unterkühlten Flüssigkeiten und Gläser," Z. Anorg. Allg. Chem. 203, 219-227.

Sokolov, A. P., 1997, "The glass transition: New ideas in an age-old field," Endeavour 21, 109-113.

Sokolov, A. P., A. Kisliuk, D. Quitmann, A. Kudlik, and E. Rössler, 1994, "The dynamics of strong and fragile glass formers: Vibrational and relaxation contributions," J. Non- 
Cryst. Solids 172, 138-153.

Soltwisch, M., A. Kisliuk, V. Bogdanov, S. Mamedov, D. Stachel, and D. Quitmann, 1999, "Brillouin scattering from transverse phonons in a molecular liquid and in an ionic salt melt mixture-A comparison," Philos. Mag. B 79, 1857-1869.

Starr, F. W., S. Sastry, J. F. Douglas, and S. C. Glotzer, 2002, "What do we learn from the local geometry of glass-forming liquids?," Phys. Rev. Lett. 89, 125501.

Stickel, F., E. W. Fischer, and R. Richert, 1995, "Dynamics of glass-forming liquids. I. Temperature-derivative analysis of dielectric relaxation data," J. Chem. Phys. 102, 6251-6257.

Stillinger, F. H., 1988, "Supercooled liquids, glass transitions, and the Kauzmann paradox," J. Chem. Phys. 88, 7818-7825.

Stillinger, F. H., 1995, “A topographic view of supercooled liquids and glass formation," Science 267, 1935-1939.

Stillinger, F. H., and P. G. Debenedetti, 2003, "Phase transitions, Kauzmann curves, and inverse melting," Biophys. Chem. 105, 211-220.

Stillinger, F. H., P. G. Debenedetti, and T. M. Truskett, 2001, "The Kauzmann paradox revisited," J. Phys. Chem. B 105, 11809-11816.

Stillinger, F. H., and T. A. Weber, 1983, "Dynamics of structural transitions in liquids," Phys. Rev. A 28, 2408-2416.

Tammann, G., 1925, "Glasses as supercooled liquids," J. Soc. Glass Technol. 9, 166-185.

Tammann, G., 1933, Der Glaszustand (Leopold Voss, Leipzig). Tammann, G., and W. Hesse, 1926, "Die Abhängigkeit der Viskosität von der Temperatur bei unterkühlten Flüssigkeiten," Z. Anorg. Allg. Chem. 156, 245-257.

Tanaka, H., 2003, "Relation between thermodynamics and kinetics of glass-forming liquids," Phys. Rev. Lett. 90, 055701.

Tarjus, G., S. A. Kivelson, Z. Nussinov, and P. Viot, 2005, “The frustration-based approach of supercooled liquids and the glass transition: A review and critical assessment," J. Phys.: Condens. Matter 17, R1143-R1182.

Teeter, M. M., A. Yamano, B. Stec, and U. Mohanty, 2001, "On the nature of a glassy state of matter in a hydrated protein: Relation to protein function," Proc. Natl. Acad. Sci. U.S.A. 98, 11242-11247.
Teodosiu, C., 1982, Elastic Models of Crystal Defects (Springer, Berlin).

Tobolsky, A., R. E. Powell, and H. Eyring, 1943, "Elasticviscous properties of matter," in Frontiers in Chemistry, edited by R. E. Burk and O. Grummit (Interscience, New York), Vol. 1, pp. 125-190.

Varotsos, P. A., and K. D. Alexopoulos, 1986, Thermodynamics of Point Defects and their Relation with Bulk Properties (North-Holland, Amsterdam).

Varshneya, A. K., 1994, Fundamentals of Inorganic Glasses (Academic, New York).

Vogel, H., 1921, "Das Temperaturabhängigkeitsgesestz der Viskosität von Flüssigkeiten,” Phys. Z. 22, 645-646.

Wales, D. J., 2003, Energy Landscapes (Cambridge University Press, Cambridge, England).

Wallace, D. C., 1972, Thermodynamics of Crystals (Wiley, New York) (Dover edition, 1988).

Walther, C., 1931, "Ueber die Auswertung von Viskositätsangaben," Erdoel Teer 7, 382-384.

Wang, W. H., 2005, "Elastic moduli and behaviors of metallic glasses," J. Non-Cryst. Solids 351, 1481-1485.

Wang, W. H., 2006, "Correlations between elastic moduli and properties in bulk metallic glasses," J. Appl. Phys. 99, 093506.

Wang, W. H., C. Dong, and C. H. Shek, 2004, "Bulk metallic glasses," Mater. Sci. Eng., R. 44, 45-89.

Widom, B., 1967, "Intermolecular forces and the nature of the liquid state," Science 157, 375-382.

Wilson, K. G., 1983, "The renormalization group and critical phenomena," Rev. Mod. Phys. 55, 583-600.

Xia, X., and P. G. Wolynes, 2000, "Fragilities of liquids predicted from the random first order transition theory of glasses," Proc. Natl. Acad. Sci. U.S.A. 97, 2990-2994.

Yamamuro, O., I. Tsukushi, A. Lindqvist, S. Takahara, M. Ishikawa, and T. Matsuo, 1998, "Calorimetric study of glassy and liquid toluene and ethylbenzene: Thermodynamic approach to spatial heterogeneity in glass-forming molecular liquids," J. Phys. Chem. B 102, 1605-1609.

Zener, C., 1942, "Theory of lattice expansion introduced by cold-work," Trans. Am. Inst. Min., Metall. Eng. 147, 361-368. 